\title{
Factorial design studies of antiretroviral drug-loaded stealth liposomal injectable: PEGylation, lyophilization and pharmacokinetic studies
}

\author{
Beeravelli Sudhakar $\cdot$ Mylangam Chaitanya Krishna • \\ Kolapalli Venkata Ramana Murthy
}

Received: 13 December 2014/Accepted: 27 January 2015/Published online: 17 February 2015

(c) The Author(s) 2015. This article is published with open access at Springerlink.com

\begin{abstract}
The aim of the present study was to formulate and evaluate the ritonavir-loaded stealth liposomes by using $3^{2}$ factorial design and intended to delivered by parenteral delivery. Liposomes were prepared by ethanol injection method using $3^{2}$ factorial designs and characterized for various physicochemical parameters such as drug content, size, zeta potential, entrapment efficiency and in vitro drug release. The optimization process was carried out using desirability and overlay plots. The selected formulation was subjected to PEGylation using $10 \%$ PEG10000 solution. Stealth liposomes were characterized for the above-mentioned parameters along with surface morphology, Fourier transform infrared spectrophotometer, differential scanning calorimeter, stability and in vivo pharmacokinetic studies in rats. Stealth liposomes showed better result compared to conventional liposomes due to effect of PEG-10000. The in vivo studies revealed that stealth liposomes showed better residence time compared to conventional liposomes and pure drug solution. The conventional liposomes and pure drug showed dose-dependent pharmacokinetics, whereas stealth liposomes showed long circulation half-life compared to conventional liposomes and pure ritonavir solution. The results of statistical analysis showed significance difference as the $p$ value is $(<0.05)$ by one-way ANOVA. The result of the present study revealed that stealth liposomes are promising tool in antiretroviral therapy.
\end{abstract}

Keywords Stealth liposomes - Opsonization · Stearic stability · PEG-10000 - DSPE · Statistical optimization and mean residence time

\begin{tabular}{|c|c|}
\hline \multicolumn{2}{|c|}{ Abbreviations } \\
\hline AIDS & Immunodeficiency syndrome \\
\hline HIV & Human immunodeficiency virus \\
\hline DSPE & $\begin{array}{l}\text { 1,2-Distearoyl-snglycero-3- } \\
\text { phosphoethanolamine }\end{array}$ \\
\hline SUV & Small unilamellar vesicles \\
\hline PDI & Polydispersity index \\
\hline$\% \mathrm{EE}$ & Percent entrapment efficiency \\
\hline MLRA & Multiple linear regression analysis \\
\hline $\mathrm{CV}$ & Coefficient of variation \\
\hline$R^{2}$ & Coefficient of determination \\
\hline Adjusted $R^{2}$ & Adjusted coefficient of determination \\
\hline FTIR & $\begin{array}{l}\text { Fourier transform infrared } \\
\text { spectrophotometer }\end{array}$ \\
\hline DSC & Differential scanning calorimeter \\
\hline PVDF & Filters polyvinylidene difluoride filters \\
\hline$d f$ & Degrees of freedom \\
\hline SS & Sum of squares \\
\hline MS & Mean sum of squares \\
\hline$F$ & Fischer's ratio \\
\hline$S$ & Significant \\
\hline NS & Nonsignificant \\
\hline
\end{tabular}

B. Sudhakar $(\bowtie) \cdot$ M. C. Krishna · K. V. R. Murthy Department of pharmaceutical Technology,

A.U.College of pharmaceutical sciences, Andhra University, Visakhapatnam 530003, Andhra Pradesh, India

e-mail: sudhakarelixir@gmail.com

\section{Introduction}

In the field of pharmacy, cascades of improvements are taking place to overcast the obsolete ones to render the greatest comfort to patient. Drug delivery systems are 
being renovated by the ceaseless efforts made by the formulators in achieving the drug action with minimal side effects, low dosing and reduction in frequency of dosing and retention of drug in the blood compartment releasing in a sustained manner. Vesicular drug delivery systems are developing at a rapid pace to circumvent the inconveniences associated with conventional drug delivery approaches. Vesicular systems are playing great role for effective management of HIV and prevent its progression toward AIDS. Vesicles are water-filled colloidal particles. In general, vesicles made of natural or synthetic phospholipids along with/without cholesterol are called liposomes. The liposomal vesicular dispersions are structurally similar to the biological membranes with alternating layers of lipid and aqueous portions $(\mathrm{Li}$ et al. 1987; Poznansky and Juliano 1983; Teewodros et al. 2010).

Human immunodeficiency virus (HIV) infection, which leads to acquired immunodeficiency syndrome (AIDS), remains a serious worldwide health problem. In 2012, there were 35.3 million (32.2-38.8 million) people living with HIV. Based on the profound knowledge gained about the HIV replication cycle, several drug targets have been identified over the years and effective treatment options are currently available. The discovery of HIV protease inhibitors introduced new and effective first-line therapies for HIV/AIDS (Puneet and Sanjay 2010).

Various HIV protease inhibitors are in clinical use. Among them, Ritonavir is one of the antiretroviral protease inhibitor and the half-life $\left(t_{1 / 2}\right)$ is $3-4 \mathrm{~h}$. The oral bioavailability of ritonavir is variable due to its poor aqueous solubility. Its oral absorption is dissolution rate limited and it requires enhancement in solubility and dissolution rate for increasing its bioavailability. Therefore, novel drug delivery systems that may safely enhance the bioavailability of protease inhibitors are needed. Hence ritonavir was selected as a drug to load into the stealth liposomes using polyethylene glycol 10000 and delivered by parenteral route. In the present study, vesicles were prepared by ethanol injection method using $3^{2}$ factorial designs and their surface was modified by polymer coating method using polyethylene glycol 10000 (Josbert 2007; Lledo et al. 2007).

\section{Materials and methods}

1,2-distearoyl-snglycero-3-phosphoethanolamine (DSPE) and cholesterol were procured from Lipoid Pvt Ltd from Germany. Ritonavir and stearic acid gift samples were purchased from Hetero drugs Pvt Ltd, Hyderabad, India; polyethylene glycol 10000 was purchased from sigma Aldrich, India.
Experimental design

A $3^{2}$ randomized full factorial design was used in this study and two factors were evaluated, each at three levels; experimental trials were performed at all nine possible combinations. Amount of lipid (DSPE) was taken as the first independent variable $\left(X_{1}\right)$ and amount of cholesterol $\left(X_{2}\right)$ was selected as the second independent variables for liposomes. These variables varied at three levels, low level $(-1)$, medium level $(0)$, and high level $(+1)$. All the calculations were done at milligram level. Amount of stearic acid $(20 \mathrm{mg})$, amount of ritonavir $(20 \mathrm{mg}$ ) and final formulation volume $10 \mathrm{~mL}$ were kept constant. Vesicle size (nm) $\left(Y_{1}\right)$, \% entrapment efficiency $\left(Y_{2}\right)$ and $100 \%$ in vitro drug release at $24 \mathrm{~h}\left(Y_{3}\right)$ were selected as dependent variables. Values of variables and batch codes are shown in Tables 1 and 2 Design Expert ${ }^{\circledR}$ DX 8.0.7.1 trial version software was used for the generation and evaluation of statistical experimental design (Karimunnisa and Atmaram 2010; Karimunnisa et al. 2010; Krishnam Raju et al. 2014).

\section{Preparation of vesicular dispersions}

Ethanol injection method was used to prepare liposomes (Karimunnisa and Atmaram 2010; Karimunnisa et al. 2010; Krishnam Raju et al. 2014). An alternative method for producing single unilamellar vesicles (SUV) that avoids both sonication and exposure to high pressure is the ethanol injection technique described by Batzri and Korn. Accurately weighed amounts of DSPE, cholesterol, stearic acid and drug were taken in a beaker and dissolved in $2 \mathrm{~mL}$ ethanol with slight heating on a hot plate with temperature not exceeding $50^{\circ} \mathrm{C}$. The monophasic ethanolic drug lipid mixture was injected at the rate of $0.25 \mathrm{~mL} / \mathrm{min}$ using the 14-gauge needle into $10 \mathrm{~mL} \mathrm{pH} 7.4$ phosphate buffer (the aqueous phase) at $60^{\circ} \mathrm{C}$ under stirring at $500 \mathrm{rpm}$ (Remi magnetic stirrer) using a Teflon-coated bead. The aqueous phase immediately turned milky indicating the vesicles' formation. The system was kept under stirring up to $1-2 \mathrm{~h}$ to facilitate the removal of ethanol. The vesicular

Table $13^{2}$ full factorial design: factors, factor levels and responses Factors (independent variables) Factor levels used

Low (-1) Medium (0) High (+1)

\begin{tabular}{llll}
\hline Amount of lipid/surfactant & 30 & 60 & 90 \\
Amount of cholesterol & 15 & 30 & 45 \\
\hline
\end{tabular}

Responses (dependent variables)

$Y_{1}=$ particle size $(\mathrm{nm})$

$Y_{2}=\%$ entrapment efficiency $(\%)$

$Y_{3}=100 \%$ in vitro drug release at $24 \mathrm{~h}$ 
Table 2 Compositions of different batches of ritonavir-loaded liposomes

\begin{tabular}{lcl}
\hline Batch code & Amount of lipid $\left(X_{1}\right)$ & Amount of cholesterol $\left(X_{2}\right)$ \\
\hline L1 $(-1,-1)$ & 30 & 15 \\
L2 $(-1,0)$ & 30 & 30 \\
L3 $(-1,+1)$ & 30 & 45 \\
L4 $(0,-1)$ & 60 & 15 \\
L5 $(0,0)$ & 60 & 30 \\
L6 $(0,+1)$ & 60 & 45 \\
L7 $(+1,-1)$ & 90 & 15 \\
L8 $(+1,0)$ & 90 & 30 \\
L9 $(+1,+1)$ & 90 & 45
\end{tabular}

dispersions were made to $10 \mathrm{~mL}$ with the $\mathrm{pH} 7.4$ phosphate buffer and transferred to vials. The dispersion was refrigerated for $2 \mathrm{~h}$ for effective vesicle sealing. They were assigned batch numbers from $\mathrm{L} 1$ to $\mathrm{L} 9$ and stored at $2-8{ }^{\circ} \mathrm{C}$. The volume of liposomal dispersions prepared in each trial was $10 \mathrm{~mL}$ to contain $20 \mathrm{mg}$ of ritonavir.

\section{Characterization of vesicular systems}

The prepared vesicular systems were characterized for percent drug content, $\mathrm{pH}$, particle size, polydispersity index and zeta potential, percent entrapment efficiency and in vitro drug release studies.

\section{Determination of percent drug content}

One milliliter of suspension was pipetted from the ritonavir-loaded liposomal and lysed with methanol. It was further diluted with $\mathrm{pH} 7.4$ phosphate buffer and the samples were analysed spectrophotometrically at $210 \mathrm{~nm}$ for ritonavir.

Determination of $\mathrm{pH}$ of vesicular dispersions

The $\mathrm{pH}$ of the vesicular dispersions was measured by $\mathrm{pH}$ meter (Elico pH meter LI 127).

Determination of particle size, polydispersity index and zeta potential $(\zeta)$

The vesicles after dilution $(1: 100)$ with distilled water were taken in the cuvette. The cuvette was placed inside the sample holder of the instrument (Malvern Nano ZS90, Malvern, UK) for measurement of size (Prabhakar and Kishan 2011). The principle of photon correlation spectroscopy was used for determining the hydrodynamic diameter of the vesicle via Brownian motion. The observations for vesicle size were recorded at $90^{\circ}$ light scattering angle and at $25^{\circ} \mathrm{C}$. The $\zeta$ was measured based on the electrophoretic mobility of vesicle which used the Helmholtz-Smoluchowski equation.

Determination of percent entrapment efficiency (\%EE)

The $\% \mathrm{EE}$ of the vesicles was determined using ultracentrifugation technique (Karimunnisa and Atmaram 2010; Karimunnisa et al. 2010). $4 \mathrm{~mL}$ volume of vesicular dispersion was centrifuged at $15,000 \mathrm{rpm}$ for $3 \mathrm{~h}$ at a controlled temperature of $4{ }^{\circ} \mathrm{C}$ (Remi cooling centrifuge). Supernatant containing unentrapped drug was withdrawn and measured UV spectrophotometrically at $210 \mathrm{~nm}$ against $\mathrm{pH} 7.4$ phosphate buffer. All the determinations were made in triplicate. The amount of drug entrapped in liposomes was determined by Eq. 1 .

$\% \mathrm{EE}=\left[\left(C_{\mathrm{d}}-C\right) / C_{\mathrm{d}}\right] \times 100$

where $C_{\mathrm{d}}$ is the concentration of total drug and $C$ is the concentration of entrapped drug.

In vitro drug release studies by dialysis

The In vitro release studies were performed by dialysis process (Prabhakar and Kishan 2011; Narashimhan et al. 2012). Cellulose dialyzing membrane (dialysis membrane 60 from HiMedia, Mumbai, India whose molecular cut-off is $6000 \mathrm{D}$ ) was soaked in $\mathrm{pH} 7.4$ phosphate buffer overnight. $2 \mathrm{~mL}$ of vesicular dispersion equivalent to $4 \mathrm{mg}$ of ritonavir was added by tying one end of the dialysis membrane and hanging into a beaker containing $500 \mathrm{~mL}$ of $\mathrm{pH} 7.4$ phosphate buffer maintained at $37 \pm 0.5^{\circ} \mathrm{C}$ on a temperature-controlled magnetic stirrer and stirred at $400 \mathrm{rpm}$ with Teflon-coated bead. $5 \mathrm{~mL}$ aliquots were withdrawn at predetermined time intervals from the beaker and were replaced with an equal volume of fresh buffer maintained at the same temperature. The samples were analysed spectrophotometrically at $210 \mathrm{~nm}$ for ritonavir.

Statistical analysis of the data and optimization

Response surface modelling and evaluation of the quality of fit of the model for the current study were performed employing Design Expert ${ }^{\circledR}$ DX 8.0.7.1 trial version software (Dhiman et al. 2008; Huang et al. 2005; Patil and Sawant 2008). Polynomial models including linear, interaction and quadratic terms were generated for all the response variables using multiple linear regression analysis (MLRA). A second-order polynomial equation that describes the effect of independent factors on the response is expressed in the following forms: 
Linear model: $Y=\beta_{0}+\beta_{1} X_{1}+\beta_{2} X_{2}$

2FI (interaction)model: $Y=\beta_{0}+\beta_{1} X_{1}+\beta_{2} X_{2}+\beta_{12} X_{1} X_{2}$

Quadratic model $=\beta_{0}+\beta_{1} X_{1}+\beta_{2} X_{2}+\beta_{12} X_{1} X_{2}+\beta_{11} X_{1}^{2}$ $+\beta_{22} X_{2}^{2}$

where $Y$ is the dependent variable; $\beta_{0}$ is the arithmetic mean response of the nine runs and $\beta_{i}\left(\beta_{1}, \beta_{2}, \beta_{12}, \beta_{11}\right.$ and $\left.\beta_{22}\right)$ is the estimated coefficient for the corresponding factor $X_{i}\left(X_{1}, X_{2}, X_{1} X_{2}, X_{1} X_{1}\right.$ and $\left.X_{2} X_{2}\right)$. The main effects $\left(X_{1}\right.$ and $\left.X_{2}\right)$ represent the average result of changing one factor at a time from its low to high value. The interaction terms $\left(X_{1} X_{2}\right)$ show how the response changes when two factors are simultaneously changed. The polynomial terms $\left(X_{1}^{2}\right.$ and $\left.X_{2}^{2}\right)$ are included to investigate nonlinearity. The equations enable the study of the effects of each factor and their interaction over the considered responses. The polynomial equations were used to draw conclusions after considering the magnitude of coefficients and the mathematical sign they carry, i.e. positive or negative. A positive sign signifies a synergistic effect, whereas a negative sign stands for an antagonistic effect. The best fitting mathematical model was selected based on the comparisons of several statistical parameters, including the coefficient of variation $(\mathrm{CV})$, the coefficient of determination $\left(R^{2}\right)$, adjusted coefficient of determination (Adjusted $R^{2}$ ) and the predicted residual sum of square (PRESS), provided by Design Expert software. Among them, PRESS indicates how well the model fits the data and for the chosen model it should be small relative to the other models under consideration. Level of significance was considered at $p<0.05$. Mathematical relationships in the form of polynomial equations are generated using multiple linear regression analysis (MLRA) and used to find out the relative influence of each factor on the response. Analysis of variance (ANOVA) for the responses was performed to identify significant effect of factors on responses and the model parameters were obtained. The relationship between the dependent and independent variables was further elucidated using contour and response surface plots. Two-dimensional contour plots and three-dimensional response surface plots resulting from equations were obtained by the Design Expert software. These plots are very useful in study of the effects of two factors on the response at one time and predict the responses of dependent variables at the intermediate levels of independent variables. Subsequently, a numerical optimization technique by the desirability and graphical optimization technique by the overlay plot approach were used to generate the new formulation with the desired responses. An optimized formulation was developed by setting constraints (goals) on the dependent and independent variables. To validate the chosen experimental design, the resultant experimental values of the responses were quantitatively compared with those of the predicted values and calculated the percent relative error by the following Eq. 5 .

$$
\begin{aligned}
\% \text { Relative error }= & \frac{(\text { Predicted value }- \text { Experiment value })}{\text { Predicted value }} \\
& \times 100 \quad \text { P }
\end{aligned}
$$

Preparation of stealth vesicular dispersions

One milliliter $10 \% \mathrm{w} / \mathrm{v}$ of polyethylene glycol 10000 polymeric aqueous solution was used for the preparation of stealth liposomes. Preparation was done by injecting $1 \mathrm{~mL}$ of $10 \% \mathrm{w} / \mathrm{v}$ of polyethylene glycol 10000 to the vesicular dispersions that was being stirred at $500 \mathrm{rpm}$ slowly to ensure uniform coating of PEG around the vesicles (Minghuang et al. 2009; Yang and Guangji 2008; Yang et al. 2007).

\section{Characterization of stealth vesicular dispersions}

The prepared stealth vesicles were characterized for percent drug content, $\mathrm{pH}$, particle size, \%EE, zeta potential and in vitro drug release by dialysis as discussed above. The in vitro drug release profile of stealth vesicular dispersions was fitted to release kinetic models.

Transmission electron microscopic studies of stealth vesicles

A drop of stealth vesicular dispersions was applied on a carbon film-covered copper grid. Excess dispersion was blotted from the grid with filter paper to form a thin film specimen. The sample was then stained with $2 \%$ uranyl acetate, air dried and examined under transmission electron microscope (Hitachi, H-7500) at a magnification of 60,000× (John and Lonnie 1998).

Fourier transform infrared spectrophotometer (FTIR) studies

Appropriate amounts of ritonavir, lipid (DSPE), cholesterol, stearic acid, and stealth liposomes were studied for interaction studies with the functional groups of the drug and other used excipients. $\mathrm{KBr}$ pressed pellet technique was used in the preparation of pellet (Karimunnisa and Atmaram 2010; Karimunnisa et al. 2010; Shilpi et al. 2010). The resultant pellet was kept in the IR chamber and 
IR spectra of the mixtures were recorded on a Bruker FTIR spectrophotometer equipped with Opus software.

\section{Differential scanning calorimetry}

Differential scanning calorimetry (DSC) is a frequently used thermoanalytical technique that generates data on melting endotherms and glass transitions. The DSC curve of the pure drug and stealth liposomes was obtained using a differential scanning calorimeter (Mettler Toledo STAR eSW 8.10, Model DSC 822e) at a heating rate of $10^{\circ} \mathrm{C} /$ min from 25 to $150^{\circ} \mathrm{C}$. An empty aluminium pan was served as reference. Nitrogen was used as a purge gas, at the flow rate of $20 \mathrm{~mL} / \mathrm{min}$ for all the studies (Chen and Yu 2002).

\section{Stability studies}

Stability studies were conducted for conventional liposomes (L5) and stealth liposomes for a period of 6 months. Stability studies were carried out on selected formulations packed in screw-capped high-density polyethylene (HDPE) amber colour bottles which were charged for long-term stability studies according to ICH guidelines $\left(2-8 \pm 2{ }^{\circ} \mathrm{C} /\right.$ $60 \pm 5 \% \mathrm{RH})$ and $\left(25 \pm 2{ }^{\circ} \mathrm{C} / 60 \pm 5 \% \mathrm{RH}\right)$ for a period of 6 months in stability chambers. The samples were withdrawn at time intervals of 0,3 and 6 months and evaluated for drug content $(\%)$, particle size, zeta potential and \%EE (Karimunnisa and Atmaram 2010; Karimunnisa et al. 2010).

\section{Lyophilization cycle}

Conventional liposomes (L5) and stealth liposomes were selected for lyophilization using Christ Alpha 1-2 LD Freeze Dryer. $5 \%$ w/v mannitol solution was used as cryoprotecting agent. The selected vesicular systems were initially filtered through $0.22 \mu \mathrm{m}$ filter under aseptic conditions in sterile area into a glass vial and $10 \mathrm{~mL}$ of each vesicular system in $\mathrm{pH} 7.4$ phosphate buffer was added with $10 \mathrm{~mL} 5 \% \mathrm{w} / \mathrm{v}$ mannitol solution in water and thoroughly mixed. The process involves two steps. The sample was cooled to $-60{ }^{\circ} \mathrm{C}$ by adjusting the condenser temperature with a pressure of 200 Torr. This primary drying process was continued for $8 \mathrm{~h}$ during which unbound water was removed completely by sublimation. The secondary drying process for removing the bound water was continued for $4 \mathrm{~h}$ at a temperature of $40^{\circ} \mathrm{C}$. The entire process of lyophilization was carried out under aseptic conditions. The product was sealed and used for in vivo studies (Sanyog et al. 2013; Ghanbarzadeh et al. 2013).
In vivo pharmacokinetic study

The study was conducted in male Wistar rats weighing in the range of 200-250 $\mathrm{g}$ following parenteral administration. The selected conventional and stealth vesicular systems were tested against the pure ritonavir solution. Before administration, the selected vesicular formulations were subjected to two-step sterilization process of filtration under aseptic conditions using sterile grade $0.22 \mu \mathrm{m}$ PVDF filters, followed by lyophilization. The conditions used in the lyophilization process were established by initial trials. The lyophilized formulations were reconstituted with previously sterilized $\mathrm{pH} 7.4$ phosphate buffer saline before administration to rats by tail vein. The pure ritonavir drug solution was subjected for sterilization using sterile grade $0.22 \mu \mathrm{m}$ PVDF filters under aseptic conditions. The animals were divided into three groups, each group containing four animals. All groups were intravenously administered via tail vein of a rat and study was carried out for a period of $24 \mathrm{~h}$. Group 1 was given pure ritonavir solution; Group 2 and Group 3 were administered with conventional liposomes and stealth liposomes. The animal dose was calculated as $2 \mathrm{mg} / 250 \mathrm{~g}$ for rat for current study. Serial blood samples $0.25 \mathrm{~mL}$ aliquots of blood samples were obtained from the rat's retro-orbital sinus and stored in microcentrifuge tubes containing dipotassium ethylene diamine tetraacetic acid. Samples were taken at 0, 0.25, 0.5, 1, 2, 4, 6, $8,10,12$ and $24 \mathrm{~h}$ post dose. Plasma was immediately separated by centrifugation at 7,500 rpm for $15 \mathrm{~min}$ from the blood samples and stored in frozen conditions at $20{ }^{\circ} \mathrm{C}$ with appropriate labelling of subject code number, study date, and collection time prior to analysis. The concentration of ritonavir in rat plasma samples was measured by the reverse-phase high-performance liquid chromatography (HPLC) (Lledo et al. 2007; Kageyama et al. 2005).

\section{Analysis of the ritonavir in rat plasma by HPLC}

Prior to injection of the sample, ritonavir was extracted from the plasma by precipitating the plasma proteins as per the following procedure: $0.1 \mathrm{~mL}$ of plasma was transferred into the Eppendorf microcentrifuge tubes. To this, $0.1 \mathrm{~mL}$ of internal standard (didanosine) and $0.2 \mathrm{~mL}$ of methanol were added and vortexed for $5 \mathrm{~min}$ in Remi cyclomixer (R24, Rimek, Mumbai, India) followed by centrifugation for $20 \mathrm{~min}$ at 7,500 rpm. After centrifugation, supernatant was collected and $20 \mu \mathrm{L}$ of the supernatant was injected into the HPLC by Hamilton microsyringe (RZ07939-01, Mumbai, India). Peak area and height were computed and the concentration of the ritonavir in the sample was determined by the standard graph (peak area ratios of ritonavir to didanosine versus concentration of ritonavir). 
Table 3 Physicochemical characterization of ritonavir vesicles

\begin{tabular}{lrrrrr}
\hline Batch code & \multicolumn{1}{c}{$\begin{array}{l}\text { \% Drug } \\
\text { content }\end{array}$} & Size $(\mathrm{nm})$ & PDI & Zeta potential $(\mathrm{mV})$ & \%EE \\
\hline L1 & $97 \pm 0.5$ & $202 \pm 0.1$ & $0.196 \pm 0.0$ & $-24 \pm 1.2$ & $36 \pm 1.5$ \\
L2 & $101 \pm 0.9$ & $160 \pm 0.2$ & $0.310 \pm 0.1$ & $-30 \pm 0.1$ & $48 \pm 1.7$ \\
L3 & $98 \pm 0.1$ & $180 \pm 0.6$ & $0.204 \pm 0.1$ & $-20 \pm 0.1$ & $32 \pm 3.0$ \\
L4 & $97 \pm 0.3$ & $90 \pm 0.1$ & $0.242 \pm 0.0$ & $-26 \pm 1.1$ & $7.4 \pm 0.3$ \\
L5 & $98 \pm 0.5$ & $49 \pm 0.3$ & $0.171 \pm 0.1$ & $-33 \pm 0.4$ & $93 \pm 1.7$ \\
L6 & $98 \pm 0.6$ & $114 \pm 0.1$ & $0.302 \pm 0.2$ & $-24 \pm 0.2$ & $7.4 \pm 1.5$ \\
L7 & $101 \pm 0.7$ & $221 \pm 0.0$ & $0.367 \pm 0.1$ & $-28 \pm 0.3$ & $7.4 \pm 0.9$ \\
L8 & $97 \pm 0.8$ & $202 \pm 0.1$ & $0.354 \pm 0.0$ & $-32 \pm 0.4$ & $52 \pm 2.0$ \\
L9 & $98 \pm 1.0$ & $228 \pm 0.0$ & $0.315 \pm 0.1$ & $-22 \pm 0.2$ & $66 \pm 1.3$ \\
\hline
\end{tabular}

A sensitive HPLC method was developed and validated in the present study to estimate the plasma concentration of drug (Lledo et al. 2007). Samples were analysed using Grace Smart Altima C-18 column $(150 \times 4.6 \mathrm{~mm}, 5 \mu)$ with mobile-phase acetonitrile: phosphate buffer $\mathrm{pH} 6.9$ $(55: 45 \% \mathrm{v} / \mathrm{v})$ at a flow rate of $1 \mathrm{~mL} / \mathrm{min}$ and detection wavelength was $235 \mathrm{~nm}$ using a Shimadzu SPD M10 AT VP model Photo Diode Array (PDA) detector (Shimadzu Corporation, Kyoto, Japan). The mobile phase was filtered through a $0.45-\mu \mathrm{m}$ membrane filter before its use. The KINETICA 4.4.1 software (Thermo Electron Corporation, UK) was used to calculate the pharmacokinetic parameters. Pharmacokinetic parameters were statistically analysed using one-way analysis of variance (ANOVA). All tests were performed at a level of significance of $(p<0.05)$.

\section{Results and discursions}

The present work focused on the development of ritonavirloaded stealth liposomal vesicular dispersions for parenteral delivery to enhance the circulation enhancement. Generally liposomes are prepared with various types of lipids (natural and synthetic)/and sterols. But in the present study, we selected the synthetic lipid DSPE which has high-phase transition temperature $\left(74^{\circ} \mathrm{C}\right)$ and short acyl chain length. Ethanol injection method was used as satisfactory and reproducible results were obtained. It was not evaluated earlier for its applicability in the liposomes for parenteral delivery.

The clinical use of ritonavir is very high in anti HIV therapy but the bioavailability of ritonavir is variable due to its poor water solubility. The major challenge in formulation of poorly soluble drugs for parenteral administration is to find an efficient preparation method resulting in a sufficiently high dose. Developments of parenteral formulations of such drugs are considerably limited in comparison to oral formulations. Parenteral administration of ritonavir is necessary in medication of HIV patients, who are incapable of receiving oral therapy for instance if they are unable to swallow due to health and physical conditions

The percent drug content of all the liposomes is tabulated in Table 3. The liposomal percent drug content was found to be in the range of 97-101\%. Percent drug content indicated that the ritonavir was uniformly distributed in vesicular dispersions and percent drug content near to $100 \%$ indicated no loss of the material during the preparation. The $\mathrm{pH}$ of liposomal vesicular dispersions was found to be around $\mathrm{pH}$ 7.4. As $\mathrm{pH}$ was within the range of blood $\mathrm{pH}$ (7.35-7.45), these formulations were suitable for parenteral drug delivery and no irritation was expected. No significant difference was found in $\mathrm{pH}$ of different formulations. This is because of dilution of the final vesicular systems with $\mathrm{pH} 7.4$ phosphate buffer. The results are shown in Table 3.

The mean vesicle size of ritonavir-loaded liposomes was found in the range of 49-228 $\mathrm{nm}$ and the polydispersity index (PDI) was in the range of $0.171-0.367$. The result indicated a profound effect of DSPE and cholesterol on the liposomal vesicles' size. The vesicle size and PDI results of all the nine batches of liposomes are tabulated in Table 3. Vesicles were all in the nanometer range with low polydispersity indicating the homogeneity of the particle size. It was strongly affected by the selected variables. The above result indicated that cholesterol has more predominant effect on liposomal vesicles' size. The behaviour of vesicles completely depended on the amount of cholesterol. Lower concentrations of cholesterol allowed uptake of water in the aqueous compartment of the liposomal vesicles increasing the aqueous volume and consequently increase the vesicle size whereas the high lipophilicity produced by higher levels of cholesterol prevented water uptake across the bilayer causing reduction in size.

The zeta potential values of liposomal dispersions were found to be in the range of -20 to $-32 \mathrm{mV}$, whereas for 
any liquid dosage form surface charge is essential for its stability. Vesicular systems exhibited a higher zeta potential value of $-32 \mathrm{mV}$ due to the surface charge imparting nature of stearic acid. The values of zeta potential showed that vesicles had sufficient charge to inhibit aggregation of vesicles due to electric repulsion. The liposomal zeta potential values are given in Table 3.

The percent entrapment efficiency (\%EE) of vesicles was determined using ultracentrifugation method. The liposomal \%EE varied from 32 to $93 \%$. The values are shown in Table 3 and Fig. 1. Assuming that the core and membrane of vesicles were saturated with medium, this would allow ritonavir to distribute throughout the vesicle. A proportional relation between the \%EE of ritonavir and the vesicle size was observed. Significant and continuous improvement in \%EE was seen with increase in cholesterol content but higher concentrations of cholesterol lead to rigidity in the vesicles which in turn decreased the \%EE. Important considerations in the entrapping efficiency of vesicles are the lipid surface charge, vesicle size, and aqueous volume of the vesicles. Basically ritonavir is a poorly water-soluble drug so it required high ratios of lipid to aqueous space.

The vesicular drug release mechanism was influenced by various physicochemical factors such as lipid/cholesterol composition, lamellarity, dispersion medium and method of preparation. The modified dialysis process was used for drug release. The study revealed that the release of the drug from the formulations depended on the relative amounts of lipid and cholesterol present.

The in vitro drug release profiles of liposomes are shown in Fig. 2. The percent drug release increased with increased concentration of lipid and at a certain level the percent release is retarded above that and the release was decreased at higher levels of cholesterol. This is because cholesterol at higher levels makes the lipid bilayers more rigid and retards the release of the drug. This was

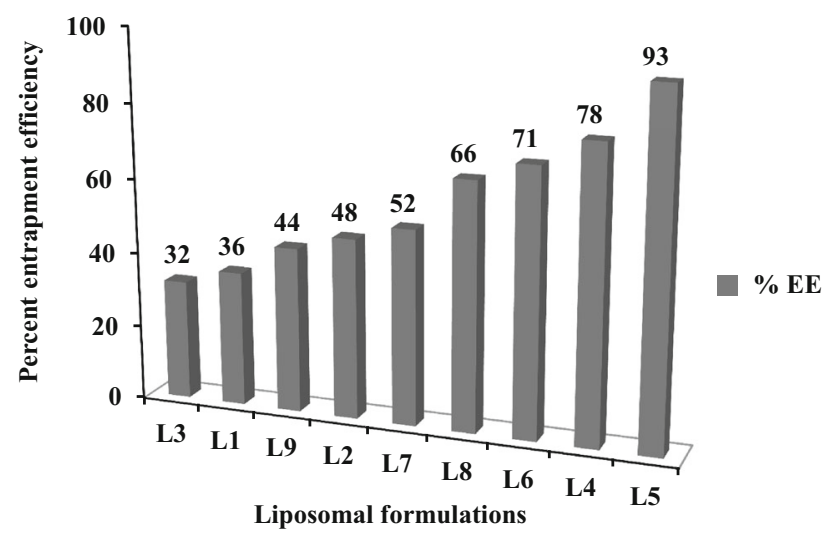

Fig. 1 Liposomal percent entrapment efficiency evident by the higher cholesterol concentration of vesicles showed around $50 \%$ of the release except (L6) formulations. The L5 formulation found to have $99 \%$ release at $24 \mathrm{~h}$. At lower concentration of lipid/cholesterol, the drug release was very less due to formation of stagnant layer.

All the vesicular systems followed first-order release kinetics, except L5 liposomal batch that followed zero order. The drug release mechanism was determined by fitting the drug release data to Higuchi and KorsmeyerPeppas equations. It was found that all the prepared liposomal vesicular dispersions followed diffusion mechanism. Plots of log fraction of ritonavir released versus log time of all the liposomal were found to be linear. The mechanism of drug release was followed by diffusion as observed from the higher ' $r$ ' value (0.9911) of Higuchi plot for selected liposomes (L5). Liposomes followed Super case II transport diffusion as indicated by ' $n$ ' value of Peppas plot (0.956) (Fig. 3).

Statistical analysis of the data and optimization

The data of the particle size and entrapment efficiency and $100 \%$ drug release at $24 \mathrm{~h}$ were fitted to quadratic polynomial model. The equations obtained are (Figs. 4, 5):

Particle size $\left(Y_{1}\right)=+52.54+39.88 X_{1} X_{1}+118.87 X_{2} X_{2}$

$\% \mathrm{EE}\left(Y_{2}\right)=+92.13-16.87 X_{1} X_{1}-34.37 X_{2} X_{2}$

$100 \%$ in vitro drug release at $24 \mathrm{~h}\left(Y_{3}\right)$

$$
=+96.96-16.38 X_{1} X_{1}-27.37 X_{2} X_{2} \text {. }
$$

From the ANOVA data, the $F$ value of all three responses for liposomes (87.70, 842.99 and 43.57) indicated that the model is significant. The $p$ value is $<0.05$ for all the response factors indicating that the models are significant. The liposomal response observation for particle size is $X_{1}, X_{1}^{2}, X_{2}^{2}, \% \mathrm{EE}, X_{1}^{2}, X_{2}^{2}$ and for $100 \%$

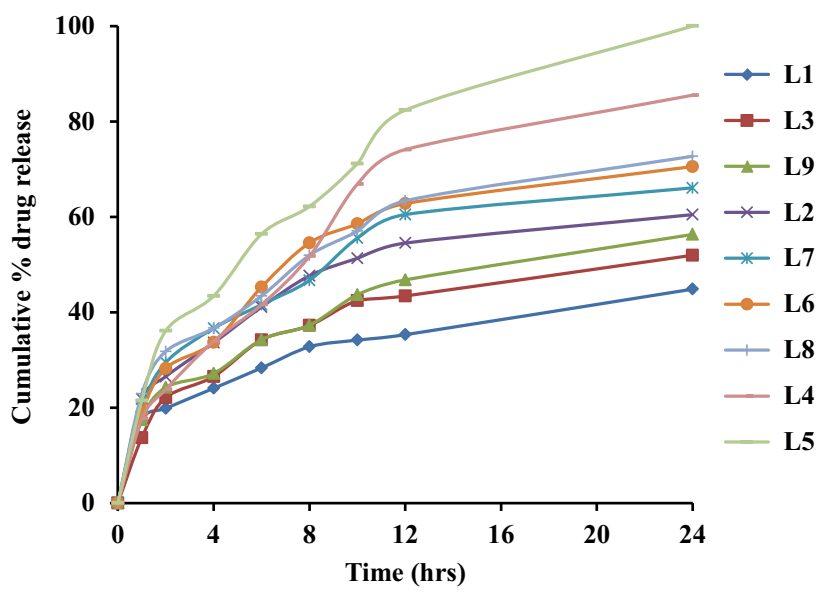

Fig. 2 Dissolution profiles of ritonavir released from liposomes

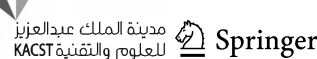


Fig. 3 Drug release kinetics and mechanism plots for L5 liposomes a zero order, $\mathbf{b}$ first order, $\mathbf{c}$ higuchi, d KorsmeyerPeppas
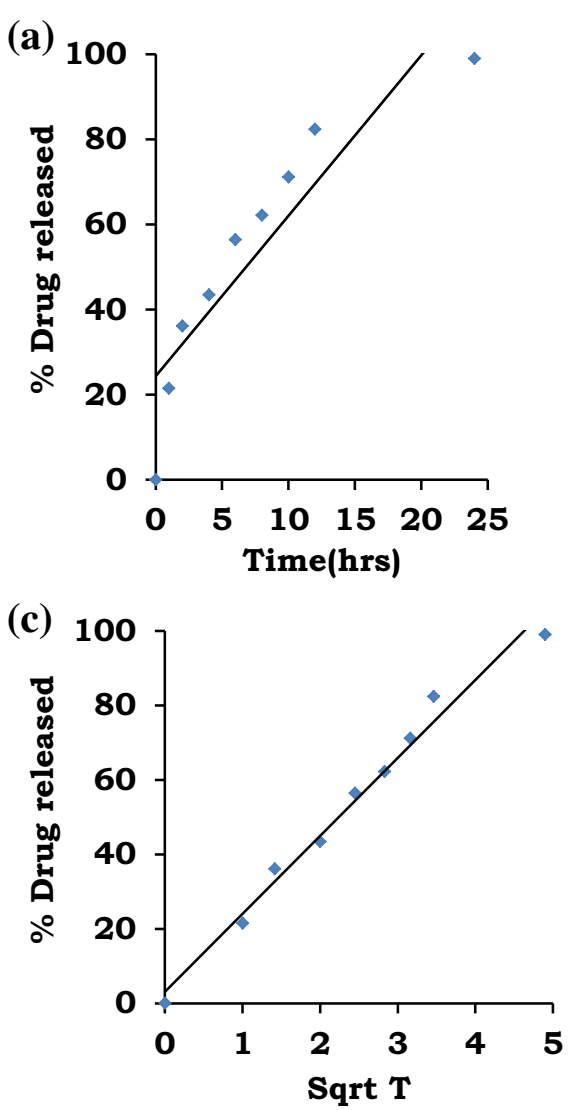

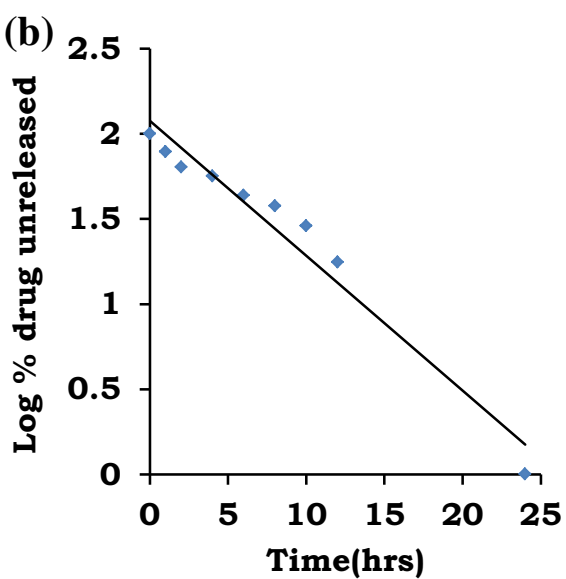

(d)

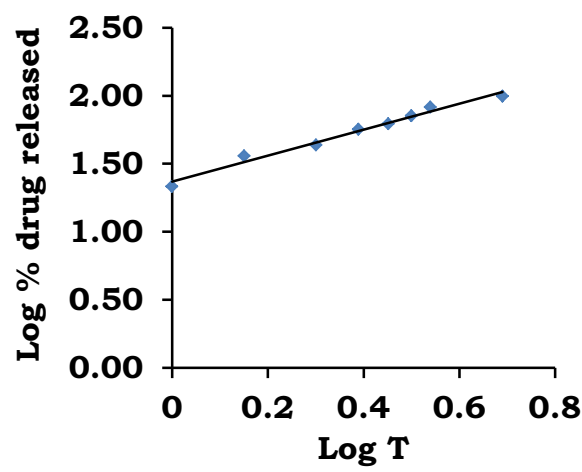

drug release $X_{1}, X_{2}, X_{1}^{2}, X_{2}^{2}$ were found to be model significant terms. The predicted $R^{2}$ values of liposomes were in reasonable agreement with the Adjusted $R^{2}$ value. The desirability and counter plots were constructed and the optimized formulae were predicted using the constraints on the dependent variables. The liposomal desirability and overlay plots are shown, respectively, in Fig. 6. The desirability function was found to be higher (near to 1) for the optimized formula indicating the suitability of the formulations. The composition of the predicted formulations was matching with L5 liposomes. However, the experiment was repeated to reconfirmed result. The \% relative error was calculated. The maximum $\%$ relative error was found to be 2.34 . However, the values were found to be $<5 \%$ and hence it confirmed the suitability of experimental design followed for this study (Tables 4, 5).

\section{Characterization of stealth vesicular dispersions}

The selected conventional liposomes' (L5) batch was subjected for PEGylation using polyethylene glycol 10000 by polymer-coating method. It is an alternative method to prepare stealth liposomes. It is a characteristic of the polymer coating method that the manner of coating is very simple, just mixing a vesicular dispersions and polymer solution, without chemically linking the polymers to the lipid molecules (Fig. 7).

The percent drug content of stealth liposomes was found to be $94.12 \%$. The mean vesicle size of stealth liposomes was found to be $116.6 \pm 0.11 \mathrm{~nm}$, respectively. The stealth liposomes' size was slightly enhanced compared to conventional liposomes due to PEG-10000 forming thick surface layer on surface of the vesicles.

Zeta potential values of stealth liposomes were found to be $-43.6 \mathrm{mV}$. The values of zeta potential showed that stealth liposomes had sufficient charge to inhibit aggregation of liposomes due to electric repulsion. When compared to conventional vesicle, stealth liposomes show more zeta potential values, due to the effect of polyethylene glycol 10000. The \%EE of stealth vesicular dispersions was slightly enhanced when compared to the conventional liposomes. PEG-10000 enhances the solubility of the drug leads to enhance the \%EE of stealth liposomes. The $\mathrm{pH}$ of stealth liposomes was also found to be around $\mathrm{pH}$ 7.4. The polyethylene glycol 10000 does not change the $\mathrm{pH}$ of the stealth vesicular dispersions and is suitable for parenteral drug delivery. The results are shown in Table 6.

The in vitro drug release profile of stealth vesicular dispersions was studied by the same method described earlier using dialysis membrane. The stealth vesicular 
Fig. 4 Contour plots for the amount of lipid (DSPE) $\left(X_{1}\right)$ and amount of cholesterol $\left(X_{2}\right)$ in liposomal formulations a on particle size $\left(Y_{1}\right)$, b on \%EE $\left(Y_{2}\right)$, c on $100 \%$ in vitro drug release at $24 \mathrm{~h}\left(Y_{3}\right)$ (a)

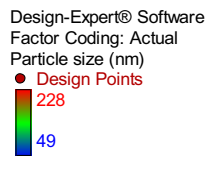

X1 = A: Lipid $\mathrm{X} 2=\mathrm{B}$ : Cholesterol

(b) Design-Expert巴 Software Factor Coding: Actual $\%$ E.E (\%)

- Design Points

32 X1 = A: Lipid
X2 = B: Cholesterol

(C) Design-Expert@ Software Factor Coding: Actual Drug release (\%) - Design Points

45

X1 = A: Lipid X2 = B: Cholesterol
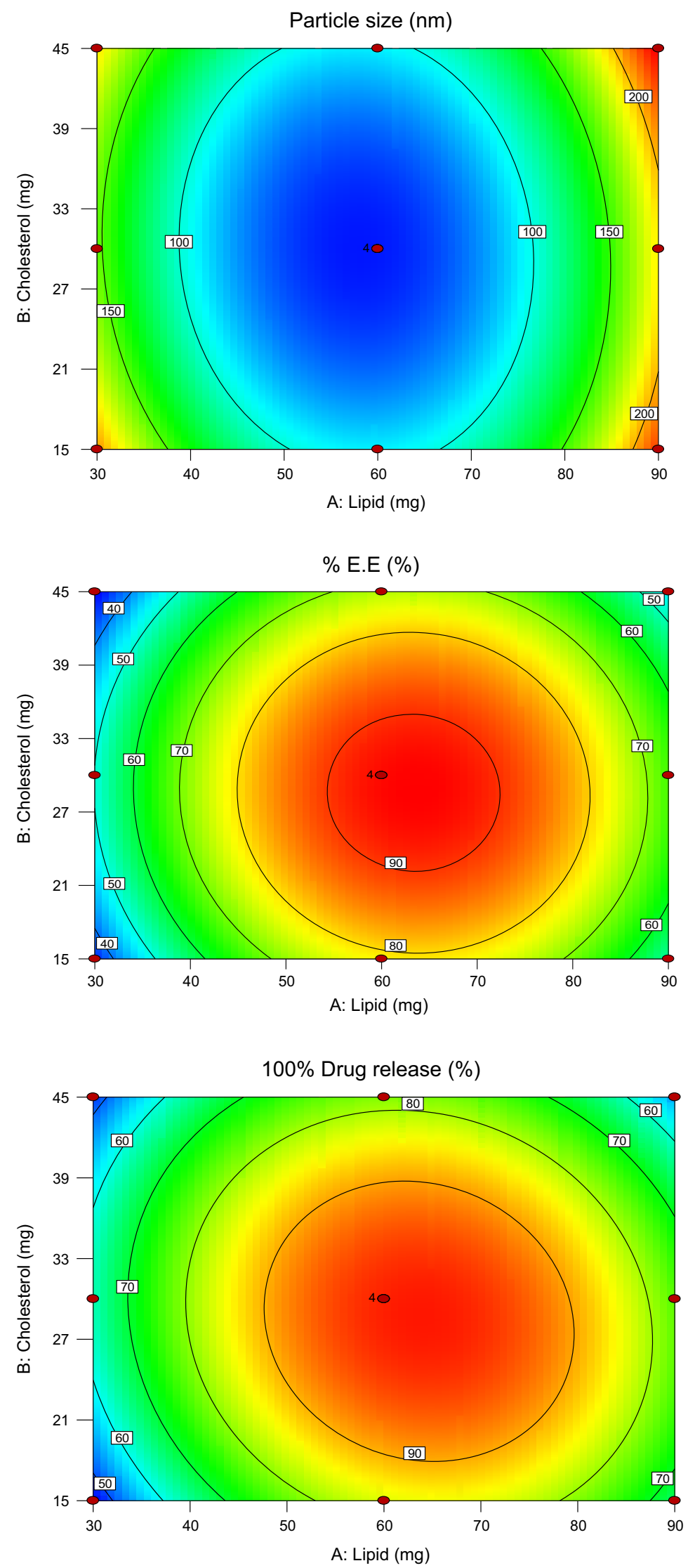

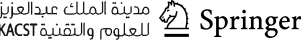


(a) Design-Expert® Software Factor Coding: Actual

Particle size $(\mathrm{nm})$

- Design points above predicted value

- Design points below predicted value

228

49

X1 = A: Lipid

X2 = B: Cholesterol

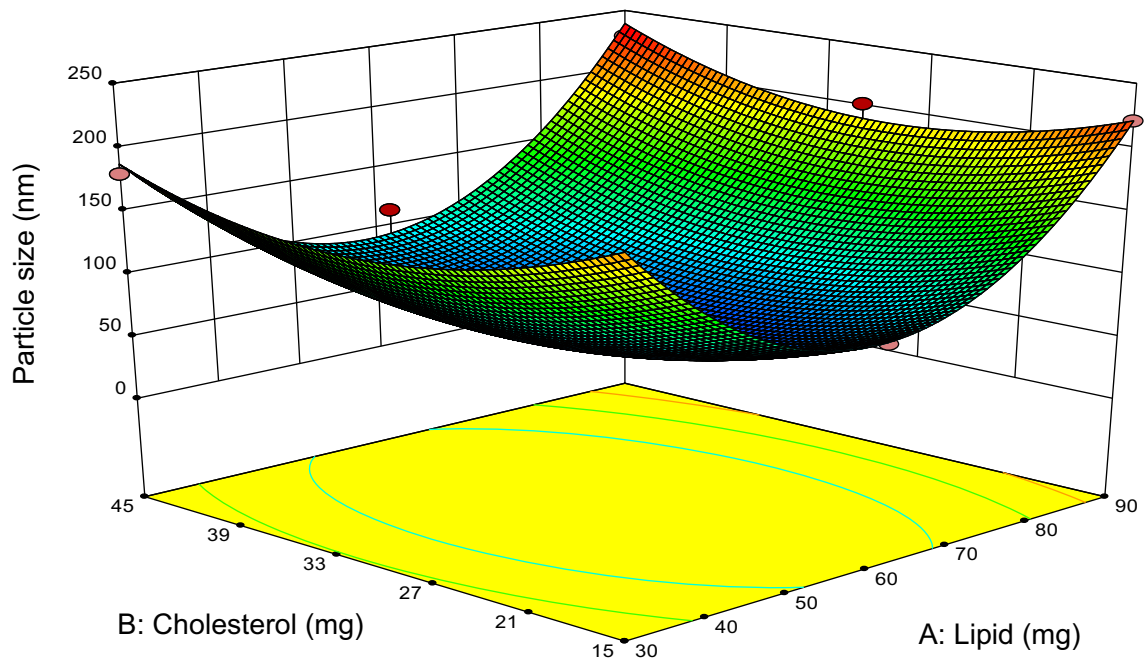

(b) Design-Expert® Software Factor Coding: Actual $\%$ E.E (\%)

- Design points above predicted value

Design points below predicted value

32

X1 = A: Lipid

X2 = B: Cholesterol

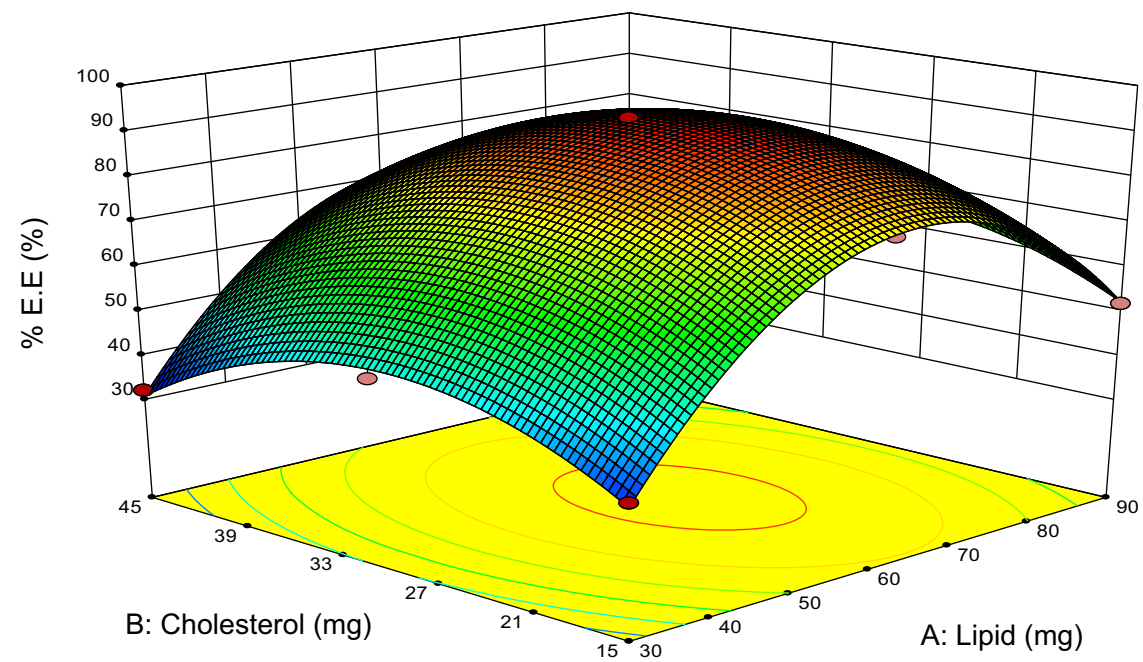

(c) Design-Expert巴 Software

Factor Coding: Actual

$100 \%$ Drug release $(\%)$

- Design points above predicted value

- Design points below predicted value

99

45

X1 = A: Lipid

X2 = B: Cholesterol

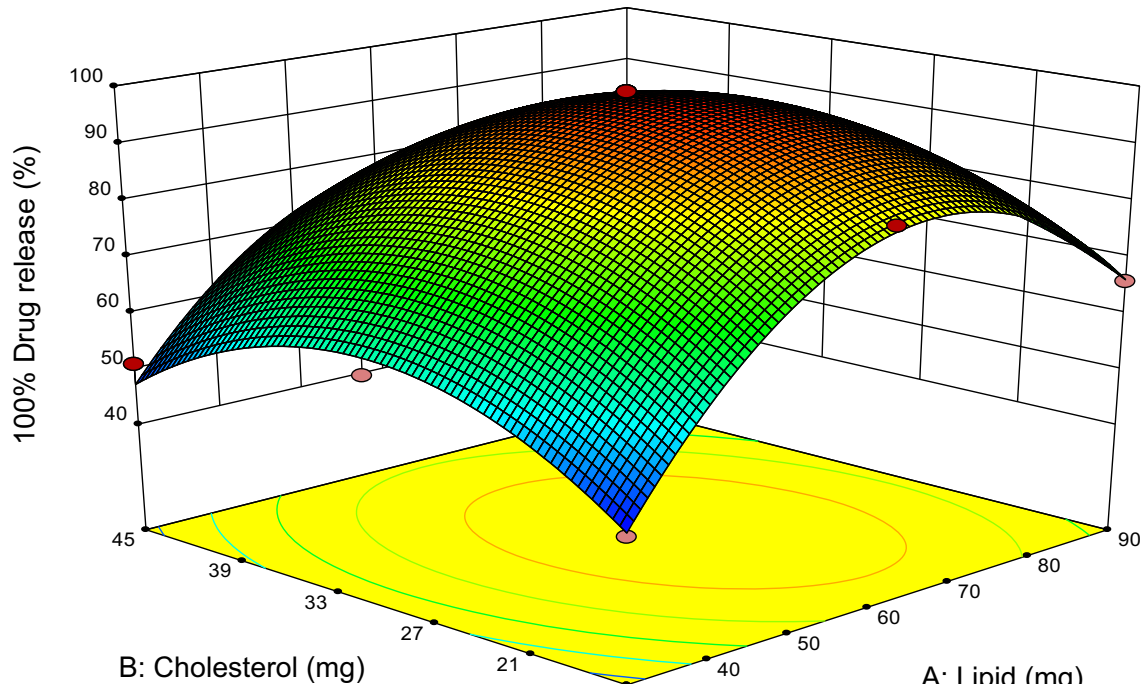

Fig. 5 Response surface plots for the amount of lipid (DSPE) $\left(X_{1}\right)$ and amount of cholesterol $\left(X_{2}\right)^{30}$ in liposomal formulations a on particle size $\left(Y_{1}\right)$, b on \% EE $\left(Y_{2}\right), \mathbf{c}$ on $100 \%$ in vitro drug release at $24 \mathrm{~h}\left(Y_{3}\right)$ 
Fig. 6 Liposomal desirability and overlay plots

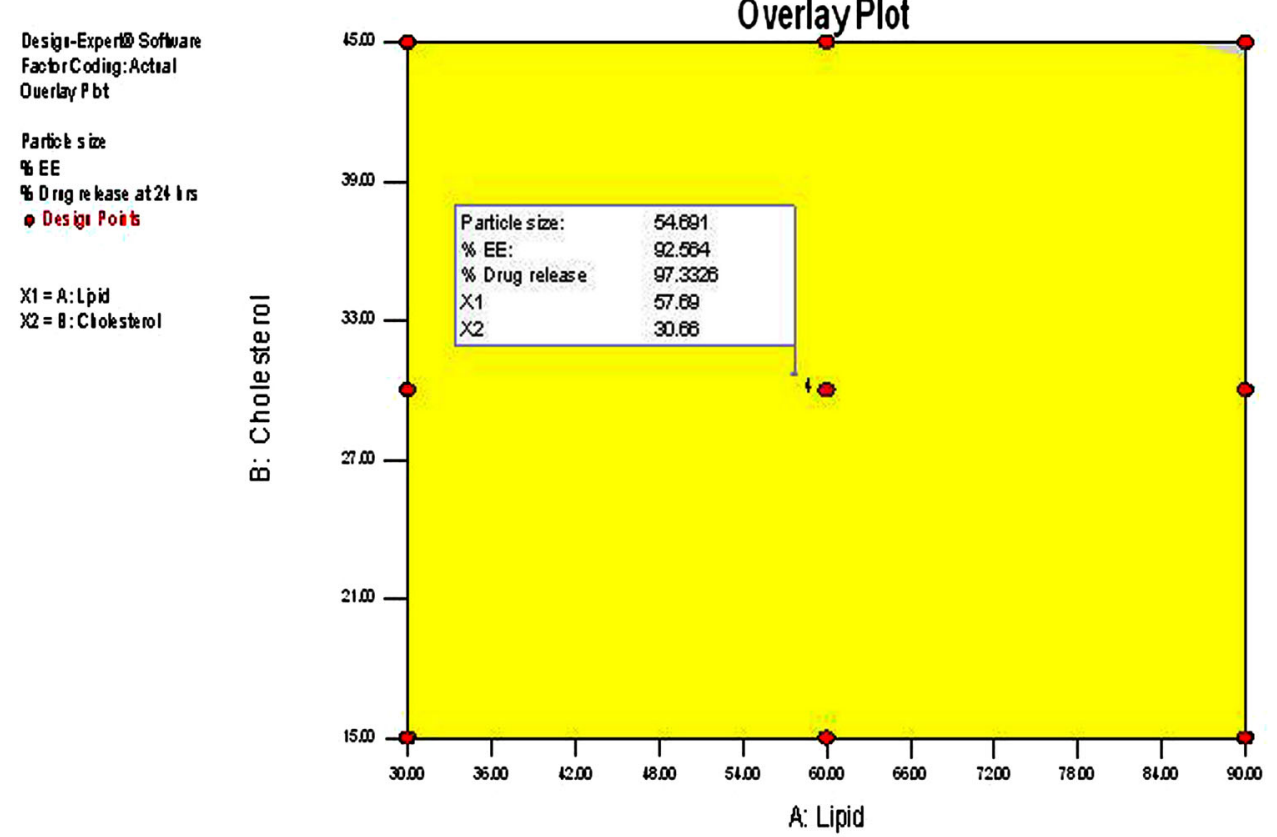

Desigu-Experto Sofluare
FacbrCoding:Actal
Oesiratily
Oes gu Poits
1000
0000

$\mathrm{X} 1=\mathrm{R}:$ Lipid $X 2=B:$ C lokst rol

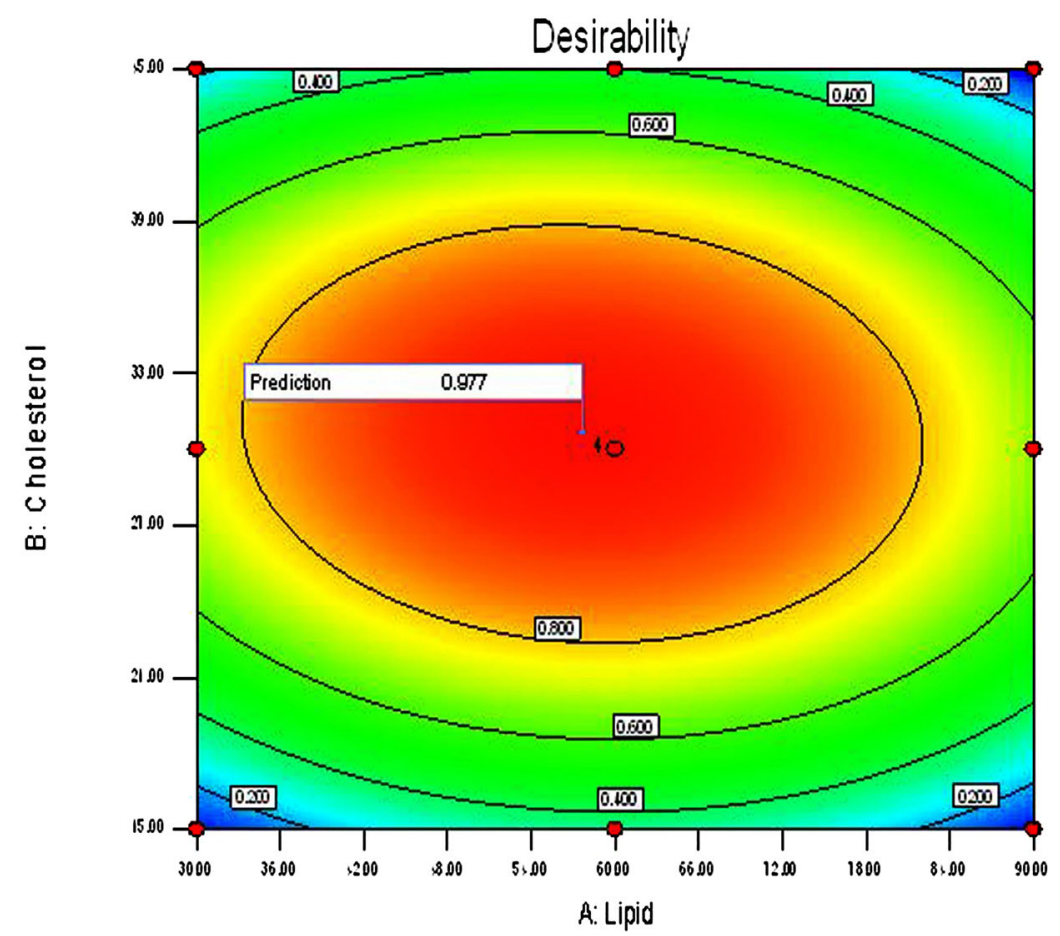

dispersions showed burst release within $1 \mathrm{~h}$ that is $17 \%$ and afterwards release was followed by diffusion manner. Due to the effect of higher molecular weight of PEG-10000 prolongs the percent drug release up to $34 \mathrm{~h}$. This is because the hydrophilic coat on the liposomes retards the drug release from stealth vesicles. Stealth liposomal dispersions followed zero-order kinetics as indicated by higher $r$ values and followed non-Fickian diffusion mechanism (Fig. 8).
FTIR studies

Fourier transform infrared spectrophotometer (FTIR) studies were performed to detect the possible interactions between drug and the excipients. The FTIR spectra of pure drug ritonavir, DSPE, cholesterol, stearic acid, PEG-10000 and stealth liposomes are shown in Fig. 9. The characteristic $\mathrm{C}-\mathrm{H}$ aliphatic stretching, $\mathrm{C}-\mathrm{H}$ or $\mathrm{C}=\mathrm{C}-\mathrm{H}$ aromatic stretching, $\mathrm{NH}$ stretching of secondary amine, $\mathrm{C}=\mathrm{C}$ 
Table 4 ANOVA for model parameters (ANOVA) for the dependent responses of liposomes

\begin{tabular}{lllllll}
\hline Parameters & SS & $d f$ & MS & $F$ & $p$ & $S$ \\
\hline $\begin{array}{l}\text { Response }\left(Y_{1}\right) \\
\text { Model }\end{array} 5,8867.46$ & 5 & $1,1773.49$ & 87.70 & $<0.0001$ & $S$ \\
$\begin{array}{l}\text { Response }\left(Y_{2}\right) \\
\text { Model }\end{array}$ & $6,000.46$ & 5 & $1,200.09$ & 842.99 & $<0.0001$ & $S$ \\
$\begin{array}{l}\text { Response }\left(Y_{3}\right) \\
\text { Model }\end{array}$ & $4,316.04$ & 5 & 863.21 & 43.57 & $<0.0001$ & $S$ \\
\hline
\end{tabular}

Table 5 Validation of predicted and optimized liposomal batch

\begin{tabular}{llll}
\hline Response & $\begin{array}{l}\text { Observed } \\
\text { Liposomes }\end{array}$ & Predicted & \% Relative error \\
\hline$Y_{1}$ & $48.9 \mathrm{~nm}$ & $50.06 \mathrm{~nm}$ & 2.31 \\
$Y_{2}$ & $94 \%$ & $95.02 \%$ & 1.07 \\
$Y_{3}$ & $98.99 \%$ & $99.84 \%$ & 0.85 \\
\hline
\end{tabular}

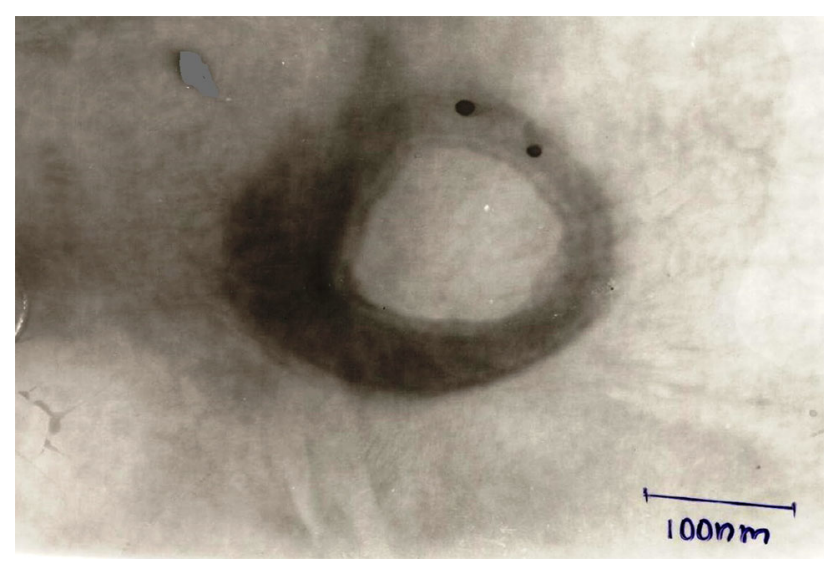

Fig. 7 Surface morphology of stealth liposomes by TEM

Table 6 Physicochemical characterization stealth vesicles

\begin{tabular}{lcc}
\hline Parameters & Stealth liposomes & Stealth niosomes \\
\hline Drug content $(\%)$ & $94.12 \pm 0.29$ & $96.24 \pm 0.47$ \\
size $(\mathrm{nm})$ & $116.6 \pm 0.1$ & $122.9 \pm 0.3$ \\
PdI & $0.193 \pm 0.08$ & $0.204 \pm 0.12$ \\
Zeta potential $(\mathrm{mV})$ & $-43.6 \pm 1.8$ & $-51.6 \pm 1.2$ \\
$\%$ EE & $96.4 \pm 1.8$ & $98.1 \pm 2.7$ \\
$\mathrm{pH}$ & $7.4 \pm 0.6$ & $7.4 \pm 0.2$ \\
\hline
\end{tabular}

Each value represents mean $\pm \mathrm{SD}(n=3)$

aromatic stretching, $\mathrm{C}=\mathrm{O}$ amide stretching, $\mathrm{C}=\mathrm{O}$ ester stretching of pure drug were observed at 2,963-2,869, $3,024-3,098,3,357,1,458-1,526,1,715$ and $1,644 \mathrm{~cm}^{-1}$ The characteristic peaks confirmed the structure of

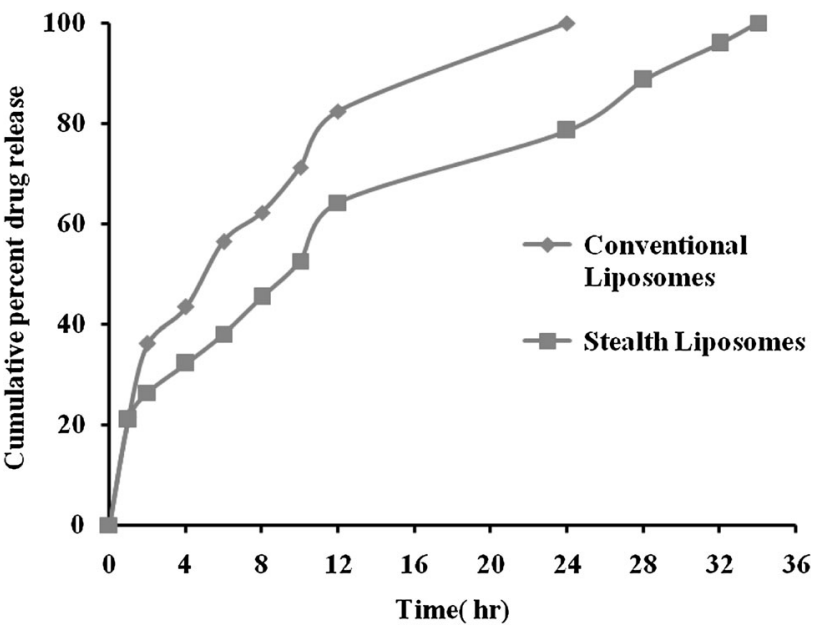

Fig. 8 In vitro comparative release profile of conventional and stealth liposomes

ritonavir. The FTIR spectrum of DSPE showed ester $\mathrm{C}=\mathrm{O}$ at $1,793.18 \mathrm{~cm}^{-1}$ and aliphatic $\mathrm{CH}$ 2,955.93$2,849.53 \mathrm{~cm}^{-1}$. The FTIR spectrum of cholesterol showed $\mathrm{C}-\mathrm{O}$ alcoholic stretching vibration at $1,022.58 \mathrm{~cm}^{-1}$, O$\mathrm{H}$ stretching vibration at $3,398.38 \mathrm{~cm}^{-1}$, aliphatic $\mathrm{C}-\mathrm{H}$ stretching vibration at $2,933.42-2,849.37 \mathrm{~cm}^{-1}$ and $\mathrm{C}=\mathrm{C}$ stretching vibration at $1,467.47$ and $1,375.42 \mathrm{~cm}^{-1}$. The FTIR spectrum of stearic acid showed $\mathrm{C}-\mathrm{H}$ aliphatic stretching vibration at $2,955 \mathrm{~cm}^{-1}$ and $\mathrm{C}=\mathrm{O}$ (acid) stretching vibration at $1,703 \mathrm{~cm}^{-1}$. The FTIR spectrum of PEG-10000 C-H aliphatic, C-O-C aliphatic ester, and OH group of PEG-10000 was observed at 2,917-2,695, 1149 , and $3,451 \mathrm{~cm}^{-1}$. All the characteristic peaks of the drug corresponding to the $\mathrm{C}-\mathrm{H}$ aliphatic stretching, $\mathrm{C}-\mathrm{H}$ or $\mathrm{C}=\mathrm{C}-\mathrm{H}$ aromatic stretching, $\mathrm{NH}$ stretching of secondary amine, $\mathrm{C}=\mathrm{C}$ aromatic stretching, $\mathrm{C}=\mathrm{O}$ amide stretching, $\mathrm{C}=\mathrm{O}$ ester stretching were present in the stealth liposomal formulations. The major peaks for the pure drug and the excipients are well in support with the theoretical prediction with respect to the functional groups. Presence of DSPE, cholesterol, PEG-10000, and steric acid did not produce any major shift in principal peaks of ritonavir and the presence of one ingredient did not produce shift in the peaks of other ingredients. This indicates that there is no interaction between drug and the excipients used in the study. Hence, FTIR spectral analysis proved the compatibility of the drug and excipients used in the study.

\section{Differential scanning calorimeter}

DSC is used to study the thermal behaviour of lipids in vesicular dispersions. Whenever these types of excipients undergo a change in physical state, such as transition from gel to liquid crystalline form, heat is absorbed. The 


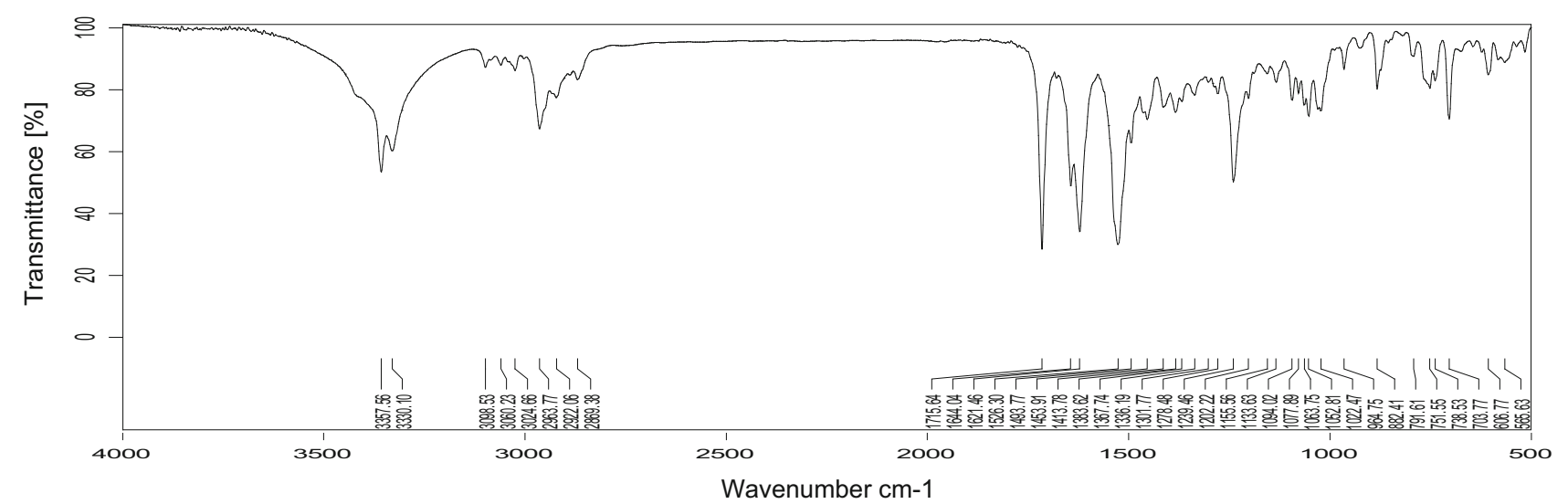

(a) Ritonavir

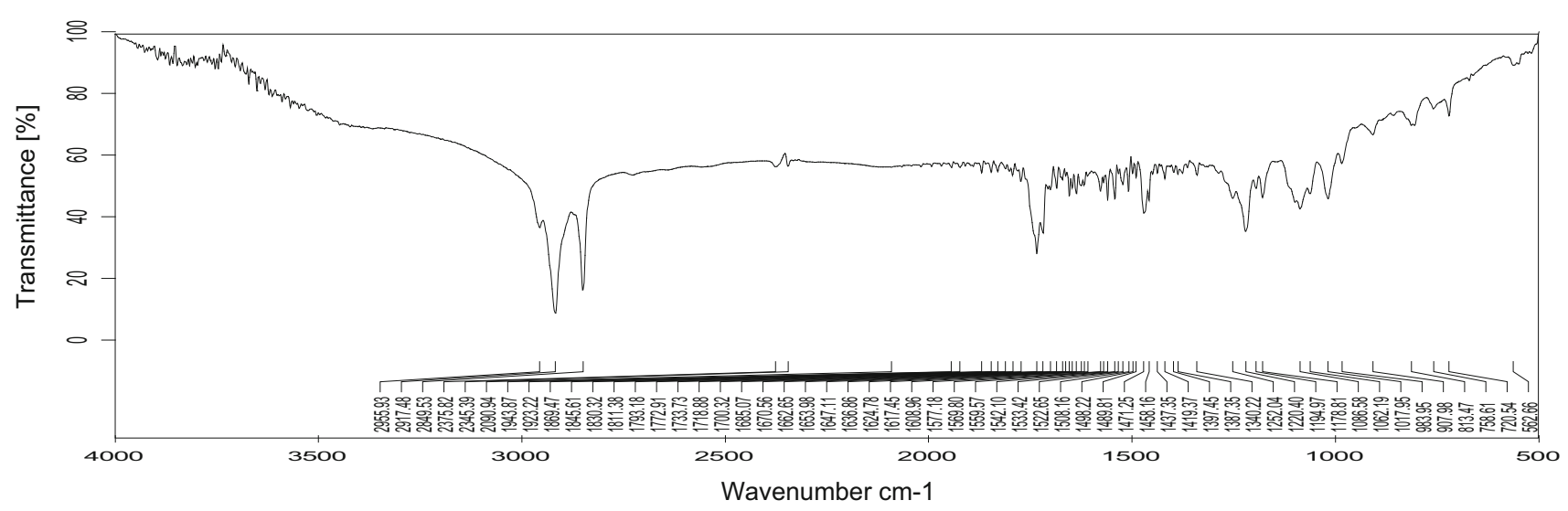

(b) DSPE

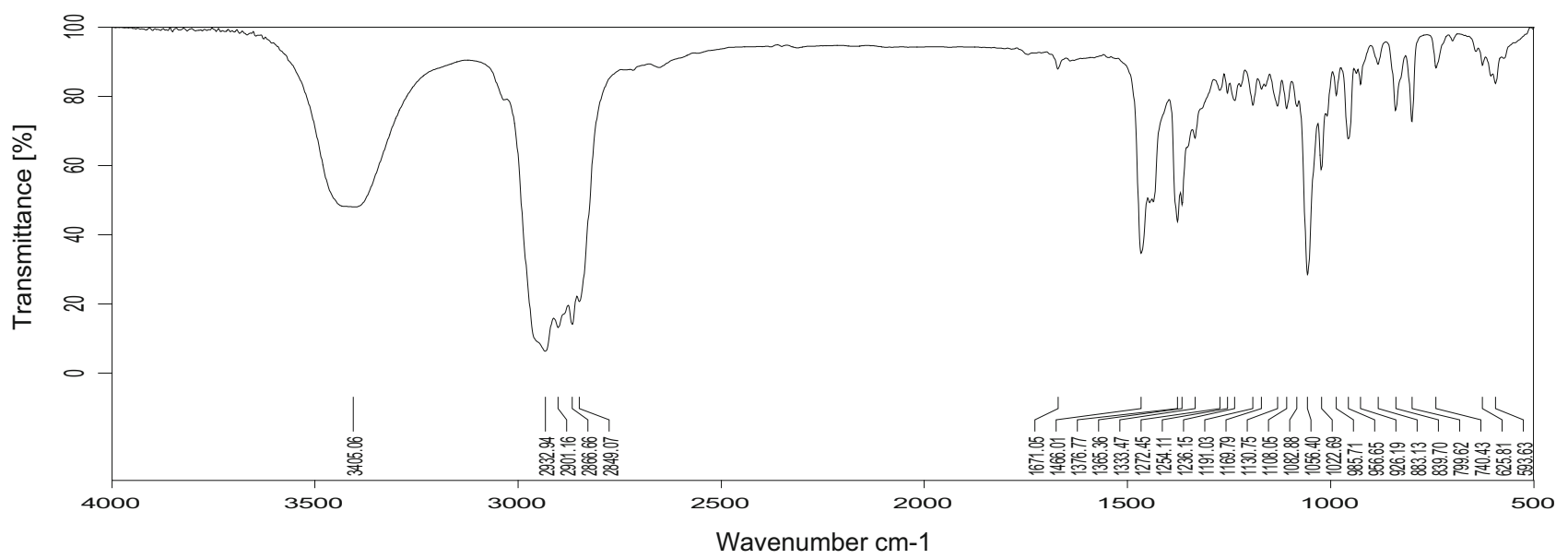

(c) Cholesterol

Fig. 9 a Ritonavir, b DSPE, c cholesterol, d stearic acid, e polyethylene glycol 10000, $\mathbf{f}$ stealth liposomes

apparatus can be used to measure heat capacity and the purity of the lipid samples. A sharp DSC peak exhibited at $124.7^{\circ} \mathrm{C}$ was correspondence to the pure drug ritonavir. A broad peak observed at $66.2{ }^{\circ} \mathrm{C}$ was correspondence to the
PEG-10000 in stealth liposomal formulation. From the DSC thermogram, it can confirm that drug was completely converted into amorphous state. So the drug peak was not observed in stealth liposomal formulation (Fig. 10). 


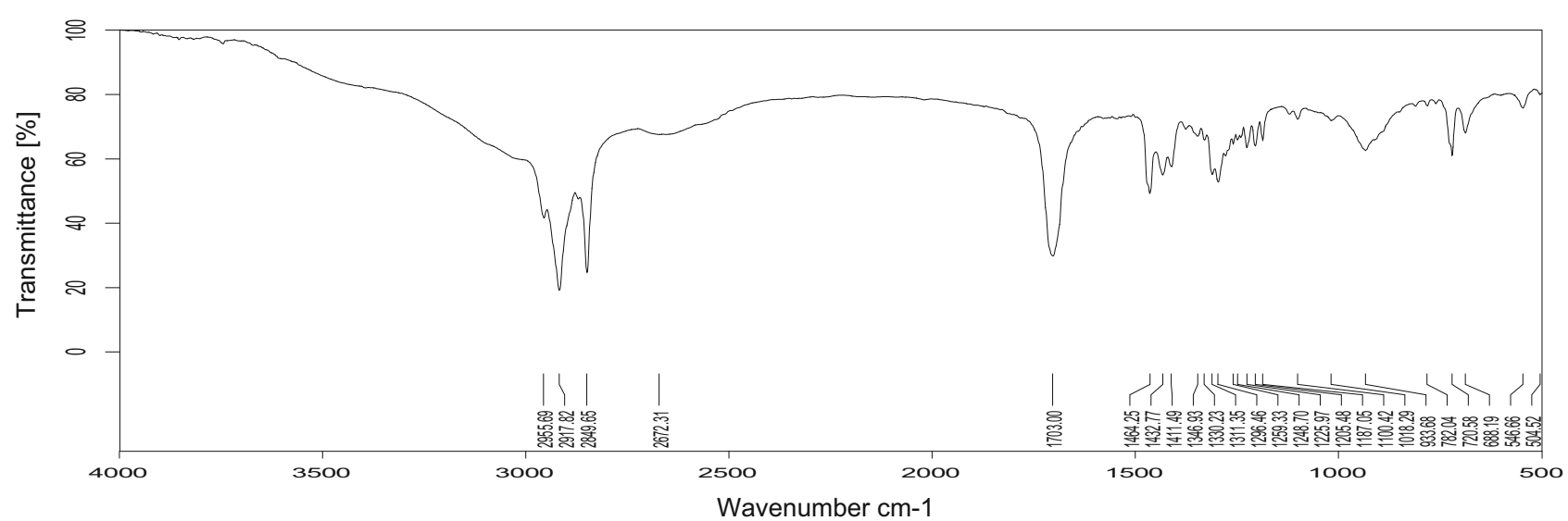

(d) Stearic acid

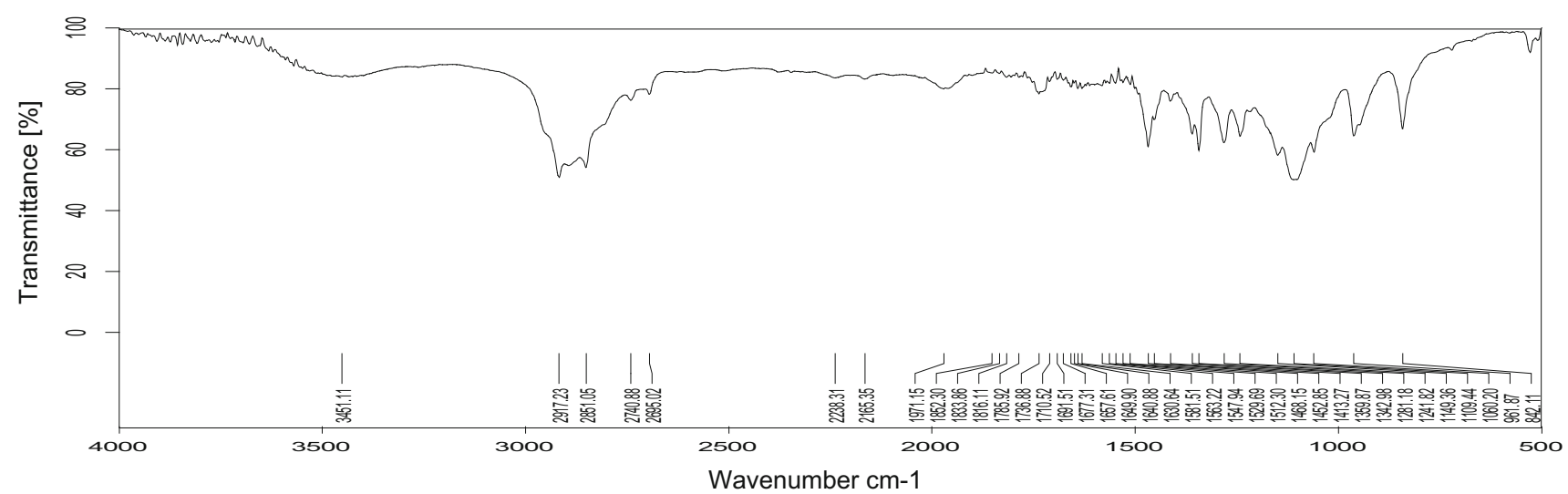

(e) Polyethylene glycol 10000

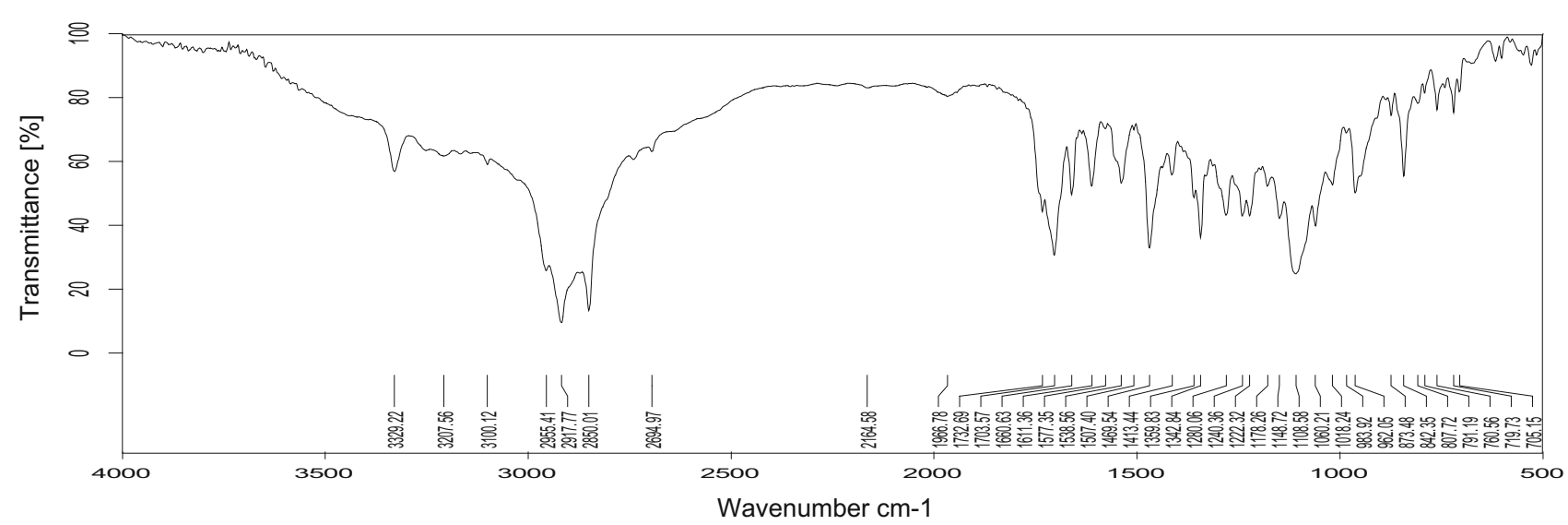

(f) Stealth liposomes

Fig. 9 continued

\section{Stability}

The stability study indicated that there are major changes observed. All the evaluated parameters were changed from the initial values. Comparatively stealth liposomes showed the better stability profile than conventional liposomes profile due to the polyethylene glycol 10000 because it enhances the surface charge of the liposomes leads enhances the stability. Aggregation and fusion of liposomes cause shift in mean size and size distribution of liposomes towards higher value and cause destabilization of vesicle, so the liposomes size therefore is a useful marker for 


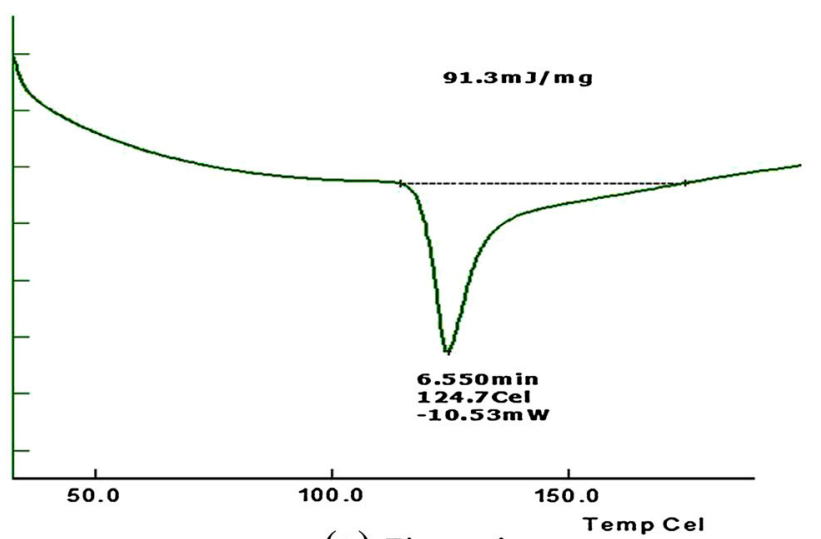

(a) Ritonavir

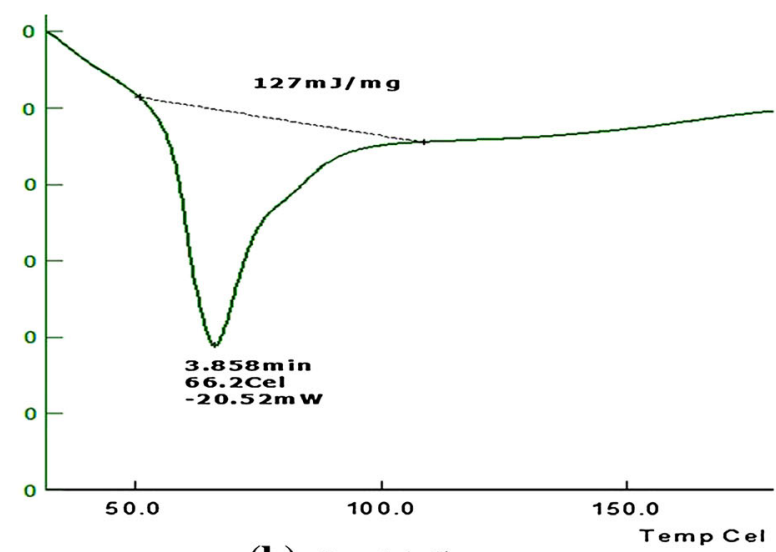

(b) Stealth liposomes

Fig.10 DSC thermograms of pure drug ritonavir and stealth liposomes

indicating changes in physical stability of vesicles. The stability studies revealed that temperature played major role, because all the vesicular dispersions are more stable at $2-8 \pm 2{ }^{\circ} \mathrm{C}$ than $25 \pm 2{ }^{\circ} \mathrm{C} / 60 \pm 5 \% \mathrm{RH}$.

In the present stability studies, results indicated that conventional liposomes are more susceptible to hydrolysis and oxidation than stealth liposomes. The values obtained revealed three main conclusions viz., firstly, there was notable difference between the stability of conventional liposomes and stealth liposomes. The stability in terms of $\%$ drug content, particle size, zeta potential and \%EE was found to be in the following stealth liposomes $>$ conventional liposomes.

In vivo pharmacokinetics

The selected formulations were subjected for the in vivo pharmacokinetic evaluations on male Wistar rats weighing around 200-250 g. The selected conventional and stealth vesicular systems were tested against the pure ritonavir solution. The selected vesicular formulations were subjected to two-step sterilization process of filtration under aseptic conditions using sterile grade $0.22 \mu \mathrm{m}$ PVDF filters, followed by lyophilization. The lyophilized products in the sealed vials were found to be free flowing without any cake formation and adherence to the wall of the container. $\mathrm{pH} 7.4$ phosphate buffer saline sterilized by autoclaving was used for the reconstitution of the lyophilized vesicular systems for parenteral administration to Wistar rats. The reconstitution time was found to be $25-30 \mathrm{~s}$ for all the products resulting in uniform dispersion of the systems with ease of flow through syringe needle. No visual damage was observed for the vesicular systems after lyophilization

From Table 7, the $\mathrm{AUC}_{0-24}$ and $\mathrm{AUC}_{0-\infty}$ of pure ritonavir solution show higher value than conventional liposomes (L5) and stealth liposomes. There was no significant difference for $\mathrm{AUC}_{0-t}$ and $\mathrm{AUC}_{0-\infty}$ among conventional and stealth liposomes. When compared to conventional liposomes (L5), stealth liposomes showed slightly higher values due to the effect of PEG-10000 stearic properties which greatly enhance long systemic circulation. The elimination rate constant $\left(K_{\mathrm{el}}\right)$ and volume of distribution $\left(V_{\mathrm{d}}\right)$ were found to be lower for stealth liposomes compared to conventional L5 and pure ritonavir solution. The $K_{\mathrm{el}}$ and $V_{\mathrm{d}}$ values further reflect the longer

Table 7 Pharmacokinetic parameters of tested products in Wistar rats

\begin{tabular}{llcrr}
\hline Parameters & Units & Pure ritonavir solution & L5 & Stealth liposomes \\
\hline$K_{\mathrm{el}}$ & $\mathrm{h}^{-1}$ & $0.412 \pm 0.019$ & $0.4299 \pm 0.09$ & $0.058775 \pm 0.005$ \\
$t_{1 / 2}$ & $\mathrm{HR}$ & $1.79 \pm 0.28$ & $1.68 \pm 0.36$ & $11.75 \pm 1.24$ \\
$\mathrm{AUC}_{0-24 \mathrm{~h}}$ & $\mathrm{ng} \mathrm{h} / \mathrm{mL}$ & $3,687.1 \pm 200.71$ & $2,887.3 \pm 209.9$ & $2,850.748 \pm 65.40$ \\
$\mathrm{AUC}_{0-\infty}$ & $\mathrm{ng} \mathrm{h} / \mathrm{mL}$ & $3,799.9 \pm 233.71$ & $2,934.07 \pm 220.5$ & $3,381.72 \pm 175.75$ \\
$\mathrm{AUMC}_{0-24}$ & $\mathrm{ng} \mathrm{h} / \mathrm{mL}$ & $12,048.7 \pm 793.7$ & $9,067.7 \pm 1,461.001$ & $17,248.98 \pm 824.22$ \\
$\mathrm{AUMC}_{0-\infty}$ & $\mathrm{ng} \mathrm{h} / \mathrm{mL}$ & $48,406.3 \pm 704.6$ & $9,989.7 \pm 1,623.5$ & $34,834.18 \pm 5,030.56$ \\
MRT $_{\text {Bioavailability }}$ & $\mathrm{h}$ & $3.5 \pm 0.12$ & $3.4 \pm 0.2$ & $11.58 \pm 1.40$ \\
Volume of distribution & $\%$ & $100 \pm 0.00$ & $77.16 \pm 1.1$ & $89.28 \pm 7.2$ \\
Clearance & $\mathrm{L} / \mathrm{ng}$ & $0.219 \pm 0.01$ & $0.223 \pm 0.04$ & $0.0306 \pm 0.002$ \\
\hline
\end{tabular}




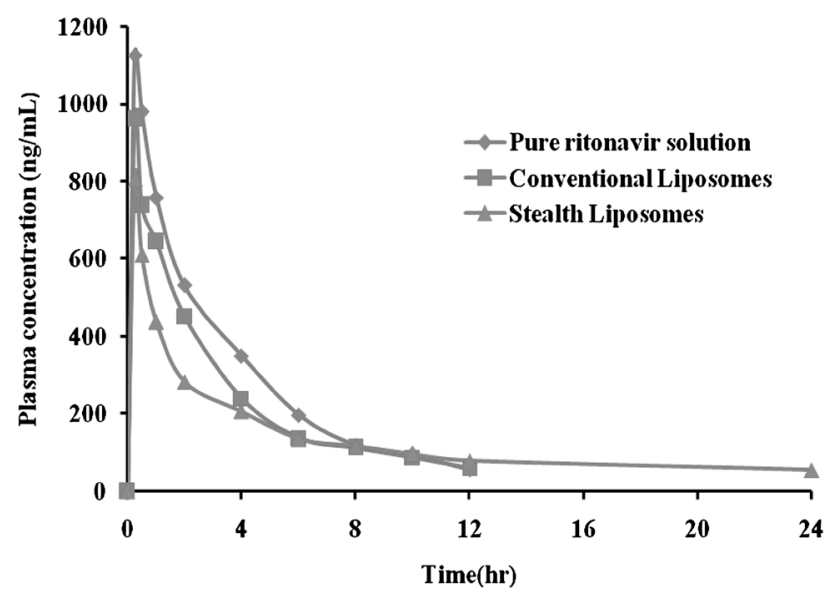

Fig. 11 Plasma concentrations of tested products in Wistar rats

blood circulating nature of stealth liposomes compared with rapidly clearing nature of conventional liposomes (L5) and pure ritonavir solution. In comparison with pure ritonavir solution, the half-life of the stealth liposomes was increased 6.65-fold and in comparison with conventional liposomes (L5) the half-life of stealth liposomes increased 6.9-fold. The lower half-life values of conventional vesicles and their pure ritonavir solution may be due to opsonization process. The Kupffer cells which are present in the liver and reticuloendothelial system (RES) are responsible to removed from the systemic circulation. The mean residence time (MRT) was found to be $11.58 \mathrm{~h}$ for stealth liposomes, while the conventional liposomes (L5) and pure ritonavir solutions found in the range of 3-3.5 h. The MRT of stealth liposomes has been increased 3.39-fold compared to conventional liposomes (L5) and pure ritonavir solution (Fig. 11).

The relative percent bioavailability $\left(F_{\text {rel }}\right)$ was found to be $100 \%, 77.1 \pm 1.1$ and $89.2 \pm 7.2 \%$ for pure ritonavir solution, conventional liposomes (L5) and stealth liposomes, respectively. Compared to pure ritonavir solution, the conventional and stealth liposomes bioavailability has been decreased because it may be the conventional liposomes rapidly cleared from systemic circulation but the stealth liposomes showed slightly higher values of relative percentage bioavailability compared to conventional liposomes (L5) due to their long systemic circulation time. The in vitro drug release of conventional and stealth vesicles showed $20 \%$ of drug release within $1 \mathrm{~h}$ because of burst release. It may be one of the reasons for the higher initial plasma drug concentration of conventional and stealth vesicles.

Based on MRT values and half-life values of the drug we can confirm that the circulation time of the drug was improved from the stealth vesicular systems compared to conventional liposomes (L5), and pure ritonavir solution. Significant difference was observed for MRT and half-life of the drug from stealth liposomes as compared to conventional liposomes (L5) and pure ritonavir solution. These in vivo pharmacokinetic results confirmed the improvements in circulation time of drug from stealth vesicular systems in the rat body.

This might be due to the effect of sterical stabilization of PEG-10000. The polyethylene glycol possessing a flexible chain occupies the space immediately adjacent to the vesicles' surface and it tends to exclude other macromolecules from this space. Consequently access and binding of blood plasma opsonins to the vesicles surface are hindered thereby inhibiting the interactions of MPS macrophages with such vesicles are inhibited. The ability of the hydrophilic shell of polyethylene glycol to avoid aggregation between vesicles and to decrease the extent of vesicle-protein interaction in biological fluids is not only due to the molecular mass of the bound polymer and its uniformity ("molecular cloud") but also due to its considerable conformational flexibility. In the present study, PEG-10000 has been used to increase the drug solubility and stability, lower the toxicity and increase the circulation time by decreasing the clearance. The ANOVA data of selected pharmacokinetic parameters $K_{\mathrm{el}}, t_{1 / 2}$, and $\mathrm{AUC}_{0-\infty}$ showed statistically significant difference between the three formulations $(p<0.05)$.

Several factors have influenced the in vivo fate of the vesicles after intravenous dosing such as particle size, surface charge, method of preparation and lipid composition. The PEGylation increased the solubility of the drug and increased its in vivo stability. The PEG-10000 shows optimum sterical stabilization property, which enhanced the systemic circulation of the ritonavir-loaded stealth vesicles and reduced the accumulation of unwanted drug actions causing toxicity. The PEGylation increased the prolong release profile of vesicles at the site of action and it leads increased the patient compliances.

\section{Discussions}

A $3^{2}$ full factorial design has been employed to produce ritonavir-loaded vesicular systems using ethanol injection method. The statistical optimization reduced the number of experiments to be carried out for obtaining formulations with desired properties. The derived polynomial equations, response and contour plots helped in predicting the values of selected independent variables for preparation of optimum vesicular systems with desired properties. Liposomes showed optimum particles size, higher \%EE, and prolong drug release for $24 \mathrm{~h}$. Among all the formulations, L5 liposomal batch showed desired optimum results such as lowest particle size, highest \%EE and maximum drug release of 99 and $96 \%$ at $24 \mathrm{~h}$. Based on optimum particle 
size, highest \%EE and maximum percent drug release, L5 batch liposomes were selected for the PEGylation. PEG10000 offers the versatile properties of flexibility, hydrophilicity, and biocompatibility. Stealth vesicular liposomes were prepared by polymer coating method. The physicochemical properties and drug release time of stealth vesicular dispersions showed better result compared to the conventional vesicular dispersions. The drug excipients' characterization parameters revealed no drug-excipients interaction.

The above in vivo studies indicated that stealth vesicular systems are suitable for lipophilic antiretroviral agent and this has been found to be suitable drug delivery systems for antiretroviral therapy. Ritonavir is a highly lipophilic antiretroviral agent and it has limited clinical use due to its poor solubility. The stealth liposomes approach altered the pharmacokinetic profile of ritonavir resulting in reduced plasma clearance and increased systemic circulation time of the drug comparatively conventional liposomes and pure ritonavir solution. The relative bioavailability was improved with longer $t_{1 / 2}$ and higher MRT and AUC values for stealth liposomes when compared to conventional liposomes. These results suggest that the ritonavir-loaded stealth liposomes could be an effective parenteral carrier for ritonavir delivery.

\section{Conclusion}

Hence the present study confirmed that the stealth vesicular systems that were prepared with various lipids/cholesterol can be used for enhanced circulation time of the drug and thereby significantly improved the drug efficiency for AIDS treatment.

\section{Conflicts of interest None.}

Open Access This article is distributed under the terms of the Creative Commons Attribution License which permits any use, distribution, and reproduction in any medium, provided the original author(s) and the source are credited.

\section{References}

Chen L, Yu Z-W (2002) Crystallization behavior of DSPE in dimethyl sulfoxide by time-resolved infrared spectroscopy and differential scanning calorimetry. J Macromol Sci Phys 41(1):137-147

Dhiman MK, Yedurkar PD, Sawant KK (2008) Buccal bioadhesive delivery system of 5-fluorouracil: optimization and characterization. Drug Dev Ind Pharm 34:761-770

Ghanbarzadeh S, Valizadeh H, Parvin ZM (2013) The effects of lyophilization on the physico-chemical stability of sirolimus liposomes. Adv Pharm Bull 3(1):25-29
Huang YB, Tsai YH, Lee SH, Chang JS, Wu PC (2005) Optimization of $\mathrm{pH}$-independent release of nicardipine hydrochloride extended-release matrix tablets using response surface methodology. Inter J Pharm 289:87-95

John JB, Lonnie DR (1998) Electron microscopy principles and techniques for biologists, 2nd edn. Janes and Bartlett Publishers, Sadbury

Josbert MM, Mastrobattista E, Gert S (2007) Liposomes for intravenous drug targeting :design and applications [Dissertation], Department of Pharmaceutics, Utrecht Institute for Pharmaceutical Sciences, Utrecht University, October 2007

Kageyama M, Namiki H, Fukushima H, Terasaka S, Togawa T, Akina T, Nobuhito S, Yukako I, Takada K (2005) Effect of chronic administration of ritonavir on function of cytochrome P450 3A and P-glycoprotein in rats. Biol Pharm Bull 28(1):130-137

Karimunnisa S, Atmaram P (2010) Liposomal delivery enhances cutaneous availability of ciclopirox olamine. Lat Am J Pharm 29(5):763-770

Karimunnisa S, Bothiraja C, Atmaram P (2010) Studies on nonionic surfactant bilayer vesicles of ciclopirox olamine. Drug Dev Ind Pharm 36(8):946-953

Krishnam Raju K, Sudhakar B, Ramana Murthy KV (2014) Factorial design studies and biopharmaceutical evaluation of simvastatin loaded solid lipid nanoparticles for improving the oral bioavailability. ISRN Nanotechnol 2014:1-8

Li VHK, Robinson JR, Lee VHL (1987) Controlled drug delivery: fundamentals and applications, 2nd edn. Marcel Dekker, New York

Lledo GR, Nacher A, Prats GL, Casabo VG, Merino SM (2007a) Bioavailability and pharmacokinetic model for ritonavir in the rat. J Pharm Sci 96(3):633-643

Lledo GR, Nacher A, Prats GL, Casabo VG, Merino SM (2007b) Bioavailability and pharmacokinetic model for ritonavir in the rat. J Pharm Sci 96(3):633-643

Minghuang H, Saijie Z, Yanyan J, Guotao T, Yuanying P (2009) Efficient tumor targeting of hydroxycamptothecin loaded PEGylated niosomes modified with transferrin. J Control Release 133:96-102

Narashimhan LR, Shilpee S, Swaminathan S, Udaykumar R, Krishnan UM (2012) Investigation on the stability of saquinavir loaded liposomes: implication on stealth, release characteristics and cytotoxicity. Int J Pharm 431(1-2):120-129

Patil SB, Sawant KK (2008) Development, optimization and in vitro evaluation of alginate mucoadhesive microspheres of carvedilol for nasal delivery. J Microencapsul 26:432-443

Poznansky MJ, Juliano RL (1983) Biological approaches to the controlled delivery of drugs: a critical review. Pharmacol Rev 36:277-336

Prabhakar K, Kishan V (2011) Brain delivery of transferrin coupled indinavir submicron lipid emulsions pharmacokinetics and tissue distribution. Colloids Surf B Biointerf 86(2):305-313

Puneet S, Sanjay G (2010) Pure drug and polymer based nanotechnologies for the improved solubility, stability, bioavailability and targeting of anti-HIV drugs. Adv Drug Deliv Rev 62:491-502

Sanyog JS, Jagadish MS, Amit KJ, Rahul RM (2013) Surfacestabilized lopinavir nanoparticles enhance oral bioavailability without coadministration of ritonavir. Nanomedicine 8(10):1639-1655

Shilpi S, Mushir A, Sanjula B, Alka A, Kumar A, Javed A (2010) Solid dispersion as an approach for bioavailability enhancement of poorly water-soluble drug ritonavir. AAPS Pharm Sci Tech 11(2):518-527

Teewodros M, Ashley EM, Nagesh K, Salvador CM, Jinjum S, Langer R, Daniel RK, Ulrich VA, Omid CF (2010) Emerging

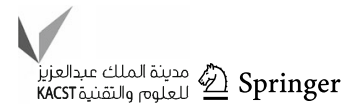


nanotechnology approaches for HIV/AIDS treatment and prevention. Nanomedicine 5(2):269-285

Yang J, Guangji W (2008) In vitro and in vivo evaluation of mPEGPLA modified liposomes loaded glycyrrhetinic acid. Int J Pharm 356:274-281
Yang T, Cui FD, Choi MK, Cho JW, Chung SJ, Shim CK, Kim DD (2007) Enhanced solubility and stability of PEGylated liposomal paclitaxel: in vitro and in vivo evaluation. Int $\mathrm{J}$ Pharm 338:317-326 